Research Paper

\title{
CD137L-macrophage induce lymphatic endothelial cells autophagy to promote lymphangiogenesis in renal fibrosis
}

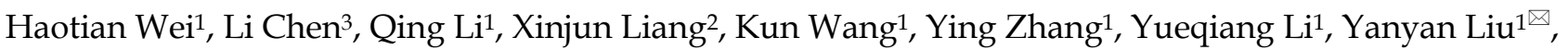 \\ Gang $X \mathbf{u}^{1}$ \\ 1. Department of Nephrology, Division of Internal Medicine, Tongji Hospital, Tongji Medical College, Huazhong University of Science and Technology, \\ Wuhan, Hubei, China \\ 2. Department of Medical Oncology, Hubei Cancer Hospital, Tongji Medical College, Huazhong University of Science and Technology, Wuhan, Hubei, China \\ 3. Department of Nephrology, Xiaogan Central Hospital, Xiaogan, Hubei, China \\ $\triangle$ Corresponding authors: Gang Xu, Department of Nephrology, Tongji Hospital, Tongji Medical College, No. 1095 Jie Fang Avenue, Wuhan, P.R. China. \\ 430030. Tel: 86-27-83663187; E-mail: xugang@tjh.tjmu.edu.cn. Yanyan Liu, Department of Nephrology, Tongji Hospital, Tongji Medical College, No. 1095 Jie \\ Fang Avenue, Wuhan, P.R. China. 430030. Tel: 86-27-69378406; E-mail: liuyy1919@163.com.
}

(C) The author(s). This is an open access article distributed under the terms of the Creative Commons Attribution License (https://creativecommons.org/licenses/by/4.0/). See http://ivyspring.com/terms for full terms and conditions.

Received: 2021.09.05; Accepted: 2021.12.19; Published: 2022.01.01

\begin{abstract}
Renal lymphangiogenesis is a new field of international nephrology in recent years and plays an important role in the progression of chronic renal disease. CDI37 was originally described as a surface molecule present on activated $T$ and NK cells and detected on hypoxic endothelial cells and inflamed blood vessels, but its function on lymphatic endothelial cells remains unclear. We investigated the relationships among CD137, lymphangiogenesis and macrophages, which are involved in interstitial fibrosis. Similar to other chronic inflammatory diseases, we found lymphangiogenesis and expression of CDI37 in the renal tissue of patients with IgA nephropathy. CD137-positive lymphatic vessels were involved in the development process of IgA nephropathy and positively correlated with serum creatinine, serum urea nitrogen, serum uric acid, and urinary $24 \mathrm{~h}$ total protein. The expression of these indicators was negatively correlated with eGFR, plasma albumin, and HB. In mouse models of UUO, we verified that CDI37 expression was significantly elevated during lymphangiogenesis and that its ligand CD137L was released by macrophages after VEGF-C stimulation in the kidney. In vitro, recombinant CD137L significantly enhanced LEC proliferation, migration and tube formation, and these effects were inhibited by CD137 siRNA. Mechanistically, the CD137L interaction with CD137 induced the transition from LC3-I to LC3-II and the expression of Atg5, Atg7, Atg12 and p62 proteins by activating the PI3K/AKT/mTOR pathway to promote autophagy. Knockdown of Atg5 and Atg7 blocked CD137L-induced autophagy. Thus, we propose that $\mathrm{CD} 137 \mathrm{~L}$ secretion by macrophages interacts with CD137 on lymphatic endothelial cells to prompt lymphangiogenesis in the kidney, which further drives fibrogenic responses. Our findings suggest that inhibition of the CD137-CD137L pathway is a novel therapeutic approach for obstructive nephropathy.
\end{abstract}

Key words: CD137L, macrophage, autophagy, lymphangiogenesis, renal fibrosis

\section{Introduction}

Renal fibrosis (RF) is the final common pathway of all chronic kidney diseases, especially the progression of IgA nephropathy (IgAN), leading to a gradual loss of renal function. In the stage of renal fibrosis, humoral immunity, hypoxia, proteinuria and acute injury cause parenchymal cell damage and the rapid recruitment of several inflammatory cells to the kidney (1-3). Then, the damaged parenchymal cells and the inflammatory cytokines and chemokines induced more different types of inflammatory cells to the lesion site. These inflammatory cells secrete a large number of cytokines and growth factors to upregulate the differentiation of renal tubular epithelial cells and endothelial cells to mesenchymal 
cells, accelerate the recruitment of bone marrow myofibroblasts, promote the activation and proliferation of inherent fibroblasts in the kidney, and lead to the development of renal fibrosis (4-6). Thus, inflammatory cells play an important "push role" either in the initial stage of renal fibrosis or in progress (7).

Evidence shows that the complex processes of inflammation are closely associated with lymphangiogenesis in some pathological conditions (8-11). Lymphangiogenesis, as an immune-related factor, regulates inflammatory cells in different organs. In inflammatory bowel disease, an increase in lymphangiogenesis indicates a good prognosis, while other forms of lymphatic alterations, including lymphangiectasia, lymphadenopathy, and lymphatic vessel occlusion, denote poor prognosis (12). During cancer progression in particular, lymphangiogenesis can exert both positive and negative effects (13). While the formation of tumor-associated lymphangiogenesis correlates with metastatic dissemination, increased severity and poor patient prognosis, the presence of functional lymphatics is regarded as beneficial for antitumor immunity and cancer immunotherapy delivery. Similarly, traditional opinion holds that lymphangiogenesis is good for clearing accumulated fluid and immune cells in kidney disease (14). The latest studies have provided insights into the role of lymphangiogenesis, which may propagate an inflammatory feedback loop, aggravating inflammation and fibrosis. Despite the explosion of knowledge on the role of lymphatic biology, several gaps remain in our understanding of the function of lymphangiogenesis in renal fibrosis.

CD137 has been shown to play a key role in T-cell-mediated responses and acts as a costimulatory molecule by binding to its ligand (CD137L) (15-17). CD137 on the surface of T helper type 1 cells (Th1) binds to CD137L expressed by macrophages or DCs and activates nuclear factor $\mathrm{\kappa B}(\mathrm{NF}-\mathrm{kB})$ by signal transduction, leading to the activation of macrophages or the maturation of DCs (18-20). Recent studies have shown that the interaction of CD137-CD137L is involved in the regulation of multiple stages of the inflammatory response and plays an important role in the cascade amplification of inflammatory responses (21-22). In renal inflammatory microenvironments, whether the CD137-CD137L signaling pathway is involved in the maintenance of inflammation and promotion of lymphangiogenesis is unknown. In this study, we aimed to identify the roles of CD137L secreted by macrophage in the development of lymphangiogenesis in a unilateral ureteral obstruction (UUO) model. In addition, we studied the relationships among $\mathrm{CD} 137^{+}$lymphatic vessels, CD137L on macrophages, and enhanced autophagy, which are all involved in interstitial fibrosis.

\section{Materials and methods}

\section{Patients}

Renal biopsy specimens were obtained from 85 patients with $\operatorname{IgA}$ nephropathy diagnosed between January 2020 and April 2020 in the Division of Nephrology, Tongji Hospital, Tongji Medical College, Huazhong University of Science and Technology. All patients met the diagnostic criteria of the Oxford Classification of IgA nephropathy 2016 (23). Patients were excluded from this study if they met the following conditions: $<18$ years of age, had a concomitant autoimmune disease, pregnancy, presence of active infection and received glucocorticoid or immunosuppressant treatment before renal biopsy. Our protocol was approved by the institutional review board or ethics committee at each center. Written informed consent was obtained from all patients.

\section{Mice}

Male C57BL/ 6 mice (age, 6-8 weeks; weight, 20$25 \mathrm{~g})$ were purchased from Beijing Vital River Laboratory Animal Center (Beijing, China). All mice were bred and maintained in specific pathogen-free (SPF) conditions at Tongji Medical College of Huazhong University of Science and Technology. Mice were anesthetized and euthanized with $1 \%$ sodium pentobarbital solution $(0.009 \mathrm{ml} / \mathrm{g}$, Sigma, USA) by i.p. injection. The renal fibrosis model was induced by unilateral ureteral obstruction (UUO) as previously reported. anti-CD137 blocking antibody (4-1BB, Clone:17B5, Bio X Cell, Lebanon NH) were injected (i.p.) at $10 \mathrm{mg} / \mathrm{kg} /$ dose twice weekly.

\section{Immunohistochemistry and immunofluorescence}

The tissues were fixed in $4 \%$ paraformaldehyde and embedded in paraffin. Antigen was recovered in citrate buffer PH 6 . The slides were blocked with 3\% H202 for $30 \mathrm{~min}$ and nonspecific antigens were blocked with serum for $30 \mathrm{~min}$. Paraffin sections were were incubated with primary rabbit anti-CD137 antibody (1:100, Abcam, USA) at $4{ }^{\circ} \mathrm{C}$ overnight. The sections were then incubated with biotinylated goat anti-rabbit Ig antibody as the secondary antibody for 30 mins, and the antibody reactions were visualized using diaminobenzidine (Gene Tech, Shanghai, China). An irrelevant isotype mouse Ig was used as the negative control.

For immunofluorescence, the sections were fixed in stationary liquid (methanol, acetone 1:1) for 10 
minutes and washed with PBST $(0.3 \%$ Tween 20 in PBS) three times. After blocking with 5\% goat serum (Zsbio, Beijing, China), the fixed tissues were incubated with the following primary antibodies: mouse anti-D2-40 antibody (Genetech, China) and rabbit anti-CD137 antibody (1:100, Abcam, USA) at $4{ }^{\circ} \mathrm{C}$ overnight. After washing with PBST, the sections were incubated with the following secondary antibodies: CY3-goat anti-rabbit IgG (1:100, Amyjet, China) and Alexa Fluor488-goat anti-mouse IgG (1:100, Amyjet, China). DAPI (1:300, Sigma-Aldrich, St Louis, MO) was used for nuclear staining. The samples were washed with PBST and mounted with fluorescence mounting medium (DAKO, Carpinteria, CA). Fluorescence signals were visualized, and digital images were obtained by a Nikon DXM 1200 digital camera and analyzed by Image-Pro Plus software in a blinded manner.

\section{Western blotting}

Kidney tissues were lysed in RIPA buffer (25 mM Tris- $\mathrm{HCl}, 150 \mathrm{mM} \mathrm{NaCl}, 1 \% \mathrm{NP}-40,1 \%$ sodium deoxycholate, $0.1 \%$ SDS, complete protease cocktail (Roche)), incubated on ice for $30 \mathrm{~min}$ and then collected by centrifugation at $12,000 \mathrm{rpm}$. for $30 \mathrm{~min}$. Approximately $20 \mathrm{ug}$ of protein lysate boiled with loading buffer was separated with SDS-PAGE electrophoresis under reducing conditions and electrotransferred onto a polyvinylidene difluoride membrane (Millipore). Following blocking with 5\% $(\mathrm{w} / \mathrm{v})$ low-fat milk in TBST $(0.1 \%$ Tween-20 in TBS) for $1 \mathrm{~h}$ at room temperature, the membrane was incubated with primary antibodies diluted in blocking buffer overnight at $4{ }^{\circ} \mathrm{C}$, followed by incubation with horseradish peroxidase-conjugated secondary antibodies for $1 \mathrm{~h}$ at room temperature. Western blotting was developed using enhanced chemiluminescence (ECL Plus; Pierce). The relative intensities of bands were quantified by ImageJ software.

\section{Flow cytometry}

The kidney tissue was weighed and put into six-well plates, and the tissue was cut with scissors, $2 \mathrm{ml}$ of $0.2 \%$ pancreatase and digested in a $37{ }^{\circ} \mathrm{C}$ thermostat for $45 \mathrm{~min}$. Tissue suspension was terminated by digestion with PBS with serum, filtrated by a strainer, and centrifuged at $1500 \mathrm{rpm}$. for 5 minutes 3 times. The cells were counted under a microscope, and the concentration of the cells in each tube was adjusted according to the count so that the cell suspension of each tube was $1 \times 10^{6}$ cells. Primary surface antibody incubations occurred for $30 \mathrm{~min}$ at $4{ }^{\circ} \mathrm{C}$. Isotype controls were added at the same concentration as primary antibodies. Samples were analyzed on a FACS Calibur (BD Biosciences) using Cell Quest software (BD Pharmingen).

The kidney tissues were weighed and put into six-well plates, and the tissues was cut with scissors and minced, digested in $2 \mathrm{ml}$ of collagenase I in a $37^{\circ} \mathrm{C}$ thermostat for $45 \mathrm{~min}$. Tissue suspension was terminated by digestion with PBS, filtrated by a strainer, and centrifuged at $1500 \mathrm{rpm}$. for $5 \mathrm{~min}$. Cells were resuspended in $1 \mathrm{~mL} 1 \mathrm{x}$ red blood cell lysis buffer, incubated for $3 \mathrm{~min}$, and washed out at $1500 \mathrm{rpm}$ for $5 \mathrm{~min}$ with PBS. The cells were counted under a microscope, and the concentration of the cells in each tube was adjusted according to the count so that the cell suspension of each tube was $1 \times 10^{6}$ cells. Primary surface antibody incubations occurred for 30 min at $4{ }^{\circ} \mathrm{C}$. The following additional antibodies were obtained from BioLegend: APC/Cy7-conjuated anti-mouse CD45 antibody, FITC-conjugated antimouse CD11b antibody, APC-conjugated anti-mouse F4/80 antibody, PE-conjugated anti-mouse CD137L antibody, BV421-conjugated Zombie dye. Samples were analyzed on a FACS Calibur (BD Biosciences) using Cell Quest software (BD Pharmingen). The data was analyzed using Flowjo v10.4 software.

\section{Real-time PCR}

For extraction of total RNA, cells were homogenized in $1 \mathrm{ml}$ TRIzol reagent, and chloroform was added to the homogenates. The mixture was incubated for $10 \mathrm{~min}$ and centrifuged at 12,000 $\mathrm{xpm}$ for $20 \mathrm{~min}$ at $4{ }^{\circ} \mathrm{C}$. Then, the chloroform was added into isopropanol. The mixture was incubated for 30 min and centrifuged at $12,000 \times \mathrm{rpm}$ for $25 \mathrm{~min}$ at $4{ }^{\circ} \mathrm{C}$. After resuspending the pullet in $75 \%$ ethanol, the mixture was centrifuged at $12,000 \times \mathrm{rpm}$ for $10 \mathrm{~min}$ twice. RNA concentration was measured by the absorbance at $260 \mathrm{~nm}$ and $280 \mathrm{~nm}$. One microgram of RNA was used for reverse transcription with a PrimeScriptTM RT reagent kit (Takara, Tokyo, Japan). The cDNA template was mixed with SYBR green PCR mix (Takara). Real-time PCR was performed using a Roche Real-Time PCR system. The following primers were used: mouse CD137 forward, CGTGCAGAA CTCCTGTGATAAC; mouse CD137 reverse, GTCCA CCTATGCTGG AGAAGG; mouse CD137L forward, CTATGGCCTAGTCGCTTTGGT, mouse CD137L reverse, CTATGGCCTAGTCGCTTTGGT; mouse gapDH forward, AGGTCGGTGTGAACGGATTTG, mouse gapDH reverse, TGTAGACCATGTAG TTGAGGTCA.

\section{CCK8 assay}

Cell proliferation was determined using the CCK-8 assay (MCE, USA). LECs were seeded and cultured in 96-well plates at a density of $10^{3}$ /well in 
$100 \mu \mathrm{L}$ medium. The cells were treated with recombinant CD137L $100 \mathrm{ng} / \mathrm{mL}$ and CD137 inhibitory for 24, 48, and $72 \mathrm{~h}$. After treatment, $10 \mu \mathrm{L}$ of CCK-8 reagent was added to each well and incubated for 2 hours away from light. The absorbance was measured at $405 \mathrm{~nm}$ using a microplate reader. All experiments were performed in triplicate.

\section{Electron microscopy}

LECs were trypsinized and pellected. The cells were fixed with $2.5 \%$ glutaraldehyde at $4{ }^{\circ} \mathrm{C}$ for $24 \mathrm{~h}$. After washing with PBS 3 times, the cells were fixed in $1 \%$ osmium tetroxide, dehydrated, and treated with propyltetrorxide, and then the cells were embedded in epoxy resin. Then, 100-nm sections were cut on an ultramicrotome and picked up on copper grids. The grids were poststained in uranyl acetate and bismuth subnitrate. Images were taken using an electron microscope (Hitachi, Japan).

\section{Cell migration assays}

Transwell assays were performed using 24-well Transwell cell culture chambers (Corning Life Science). Briefly, LECs were seeded into the top chamber at a density of $2.5 \times 10^{5}$ /well in $100 \mu \mathrm{l}$ of serum-free medium, and $750 \mu$ of cultured medium with or without recombinant CD137L protein was added to the lower chamber. After incubation for $12 \mathrm{~h}$, the migrated cells on the lower membrane surface were fixed with $4 \%$ paraformaldehyde and then stained with crystal violet. Five random fields were counted per chamber by an inverted microscope (Olympus, Japan). Each experiment was repeated three times.

\section{Wound-healing assay}

LECs were seeded in 6-well plates, grown to confluency, and then scratched across the center using a $200-\mu \mathrm{L}$ pipette tip. After rinsing with PBS three times, the medium was replenished with FBS-free DMEM to inhibit cell proliferation. Images were taken at the time of wounding $(0 \mathrm{~h})$ and at $12 \mathrm{~h}$. Migration was quantified using ImageJ software.

\section{Cell culture and transfection}

Murine bone marrow-derived macrophages (BMDMs) were flushed from the tibia and femurs of 6-week-old WT C57BL/ 6 mice. The bone marrow cells were flushed out and centrifuged for $5 \mathrm{~min}$ at $300 \times \mathrm{g}$. After eliminating erythrocytes, the remaining cells were differentiated in Dulbecco's modified Eagle's medium supplemented with $10 \%$ fetal bovine serum (Gibco, USA) with $25 \mathrm{ng} / \mathrm{mL}$ macrophage colony stimulating factor (M-CSF) for 7 days. The mouse lymph node endothelial cell line (SVEC4-10, LECS) was purchased from the China Center for Type Culture Collection (CCTCC). SVEC4-10 cells were cultured in DMEM supplemented with $10 \%$ fetal bovine serum and $1 \%$ penicillin/streptomycin. Recombinant CD137L (Cat: HY-P7446) was purchased from MedChemExpress (Shanghai, China).

LECs were transfected with control siRNA, Atg5 and Atg7 siRNA (Ribobio, China) using Lipofectamine iMAX for $24 \mathrm{~h}$. Briefly, cells were seeded into a 24-well plate. The following day the cells were transfected. Lipofectamine and siRNA were separately first mixed with OPTI-MEM at room temperature for $5 \mathrm{~min}$. Subsequently, siRNA was added to Lipofectamine-containing media, incubated for $15 \mathrm{~min}$ and then added to cells.

\section{Tube formation assay}

A pre-chilled 48-well cell culture plate was coated with $150 \mu \mathrm{L}$ of unpolymerized Matrigel (10 $\mathrm{mg} / \mathrm{mL}$ ) and incubated at $37^{\circ} \mathrm{C}$ for $1 \mathrm{~h}$. SVEC4-10 cells were harvested and seeded at a density of $10^{4}$ cells/well. The cells were incubated with or without recombinant CD137L (50 ng/mL) and anti-CD137 antibody $(5 \mu \mathrm{g} /$ well). After approximately 3 hours of incubation at $37^{\circ} \mathrm{C}$ in $5 \% \mathrm{CO} 2$, cell tube formation was assessed using an inverted fluorescent microscope.

\section{RNA sequencing and bioinformatics analysis}

For RNA-seq, total RNA was extracted from LECs. RNA isolation, library construction, and sequencing were performed on a BGISEQ-2000 (BGI, Shenzhen, China). Fold changes were calculated for all possible comparisons, and a 2-fold cutoff was used to select genes with expression changes. KEGG pathway analysis and GO-P( biological process) was performed using the $\mathrm{R}$ package, using significantly differentially expressed genes $(p<0.05)$ as target genes.

\section{mRFP-GFP-LC3 lentiviral infection}

SVEC4-10 cells were infected with lentiviral particles expressing the fusion protein mRFP-GFP-LC3 (Genomeditech, Shanghai, China) for 24 hours. Then, the transfection medium was replaced by culture DMEM supplemented with 10\% FBS for 48 h. After treatment, the cells were fixed with $4 \%$ formaldehyde. After washing with PBST 3 times, the nuclei were stained with DAPI. The cells were imaged using a confocal fluorescence microscope (Olympus, Japan).

\section{Statistical analysis}

The statistics were first analyzed for normal distribution using the Kolmogorov-Smirnov test. The Continuous variables are showed median (25th-75th percentiles) and categorical variables are showed as a 
number. Two-tailed unpaired t-tests or chi-squared tests were used for comparisons between two groups, and one-way ANOVA tests were used for three groups. Statistical analysis of data was performed using GraphPad Prism 7. P values $<0.05$ were considered statistically significant; ${ }^{*} \mathrm{P}<0.05$; ${ }^{* *} \mathrm{P}<$ $0.01 ;{ }^{* * *} \mathrm{P}<0.001, \mathrm{NS}=$ not significant.

\section{Results}

\section{CD137 expression is increased and correlated with intrarenal lymphangiogenesis and interstitial fibrosis in IgA patients}

CD137 was previously shown to play a key role in T-cell-mediated responses and acts as a costimulatory molecule by binding to its ligand (CD137L). We collected 85 clinical renal biopsy specimens from IgA nephropathy patients in our hospital from January 2020 to April 2020. There were 42 males and 43 females, aged 18 to 65 years. The diagnosis of IgA nephropathy was confirmed by renal pathological examination, and renal biopsy specimens were reassessed blindly by a single pathologist using the Oxford classification. Representative photographs of Masson's trichrome staining are shown in Figure 1A. Immunohistochemistry results showed that CD137 was strikingly expressed in proliferating mesangial cells, injured renal tubular epithelial cells, renal interstitial cells and lymphatic endothelial cells (Figure 1A). Although a previous study reported that inflamed skin contains abundant $\mathrm{CD} 137^{+}$lymphatic vessels (27), evidence of $\mathrm{CD} 137^{+}$lymphatic endothelial cells during IgA is still absent. We performed immunofluorescence staining using D2-40 as a lymphangiogenesis marker. CD137 expression was costained for D2-40, which was observed as double-positive cells in patients, while healthy individuals showed little or no costaining (Figure 1B).

To test whether the expression of CD137 in lymphatic vessels is related to the clinical parameters of IgA nephropathy patients, we first examined the quantification of colocalization of D2-40/CD137 staining, and the results were semiquantitatively analyzed with ImageJ. We collected clinical data, including age, sex, serum urea nitrogen, serum uric acid, urinary $24 \mathrm{~h}$ total protein, $\mathrm{HB}$, serum albumin, serum creatinine, and eGFR, from IgA nephropathy patients. The data analysis shows that the expression level of D2-40 was not correlated with age, sex or serum uric acid but positively correlated with serum creatinine $(\mathrm{r}=0.438, \mathrm{p}<0.001)$, serum urea nitrogen $(\mathrm{r}=0.427, \mathrm{p}<0.001)$, and urinary $24 \mathrm{~h}$ total protein $(r=0.496, p<0.001)$. The expression levels of D2-40 and CD137 colocalization areas were not correlated with age or sex but were positively correlated with serum creatinine $(\mathrm{r}=0.499, \mathrm{p}<0.001)$, serum urea nitrogen $(\mathrm{r}=0.437, \mathrm{p}<0.001)$, serum uric acid $(\mathrm{r}=0.241, \mathrm{p}=0.026)$, and urinary $24 \mathrm{~h}$ total protein $(\mathrm{r}=0.435, \mathrm{p}<0.001)$. The expression of these indicators was negatively correlated with eGFR $(\mathrm{r}=-0.473, \mathrm{r}=-0.528, \mathrm{p}<0.001)$ (Figure 1C, Table 1).

Table 1. Demographic and clinical parameters between patients with D2-40 and CD137+D2-40 expression.

\begin{tabular}{llll}
\hline Variables & ALL(n=85) & D2-40 & CD137+D2-40 \\
\hline Male gender $(\%)$ & $42.0(49.4 \%)$ & -0.151 & -0.092 \\
Age (years) & $34.0(28.0-44.0)$ & -0.053 & -0.075 \\
Serum Creatinine $(\mu \mathrm{mol} / \mathrm{L})$ & $119.0(83.0-189.0)$ & $0.438^{c}$ & $0.499^{c}$ \\
Serum urea nitrogen $(\mathrm{mmol} / \mathrm{L})$ & $6.64(4.65-9.95)$ & $0.427^{c}$ & $0.437^{\mathrm{c}}$ \\
Serum uric acid $(\mu \mathrm{mol} / \mathrm{L})$ & $375.0(325.0-454.0)$ & 0.183 & $0.241^{\mathrm{a}}$ \\
eGFR $(\mathrm{ml} / \mathrm{min} / 1.73 \mathrm{~m} 2)$ & $54.2(36.15-90.25)$ & $-0.473^{\mathrm{c}}$ & $-0.528^{\mathrm{c}}$ \\
Proteinuria $(\mathrm{g} / 24 \mathrm{~h})$ & $1.0(0.59-2.49)$ & $0.496^{\mathrm{c}}$ & $0.435^{\mathrm{c}}$ \\
Plasma albumin & $40.1(35.8-41.95)$ & $-0.284^{\mathrm{b}}$ & $-0.248^{\mathrm{a}}$ \\
HB $(\mathrm{g} / \mathrm{dL})$ & $123.5(106.25-142.5)$ & $-0.352^{\mathrm{b}}$ & $-0.327^{\mathrm{b}}$ \\
\hline
\end{tabular}

Data are presented as a percentage or median ( $25^{\text {th }}$ - 75 th percentiles).

Values of the lymphatic vessels are presented as the number of cells per millimeter squared. $\mathrm{a}<<0.05 ; \mathrm{b}<<0.01 ; \mathrm{c}<<0.001$.

eGFR: estimated glomerular filtration rate. HB: hemoglobin.

Moreover, we assessed the relationship between the Oxford classification and the density of CD137-positive lymphatic vessel angiogenesis or total lymphatic vessel angiogenesis. We found that the patients with higher scores for tubular atrophy/interstitial fibrosis showed a higher density of lymphatic vessels. However, the density of CD137-positive lymphatic vessels was better at differentiating the divergence of $\mathrm{T} 1$ and $\mathrm{T} 2$. In terms of segmental sclerosis, both the density of total lymphatic vessels and CD137-positive lymphatic vessels showed a significant association (Figure 1D).

We further investigated the potential effect of D2-40 and CD137 colocalization area, and ROC curve analysis was used to compare the specificity and sensitivity to predict fibrosis in IgA patients. With the cutoff value of $2.2 \%$ in the cohort, patients with a high percentage of colocalization areas exhibited severe tubular interstitial fibrosis (T2, 52.6\% versus 5.5\%; $\mathrm{P}<0$. 001). There was no difference in mesangial hypercellularity (M1), endocapillary hypercellularity (E1), segmental glomerulosclerosis (S1) or crescents (C2) between the two groups (Table 3). Altogether these results revealed that CD137-positive lymphatic vessels have a close relationship with the prognosis of IgAN. 
A
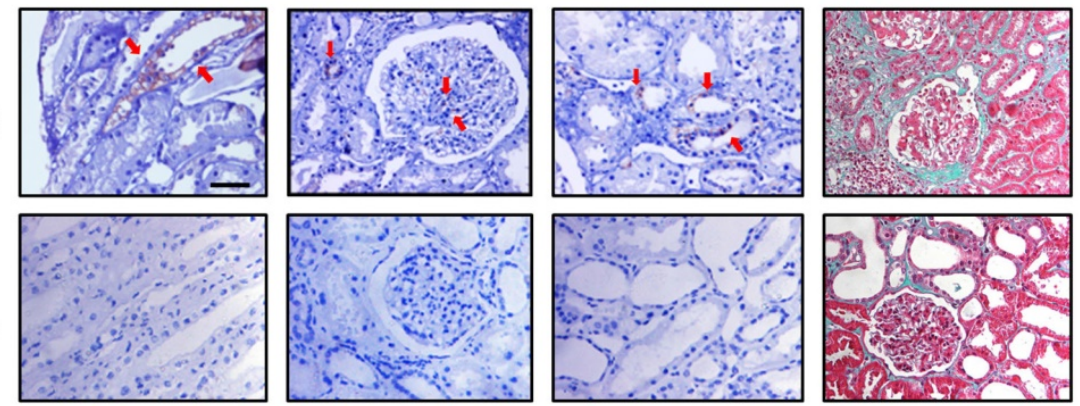

B

D2-40
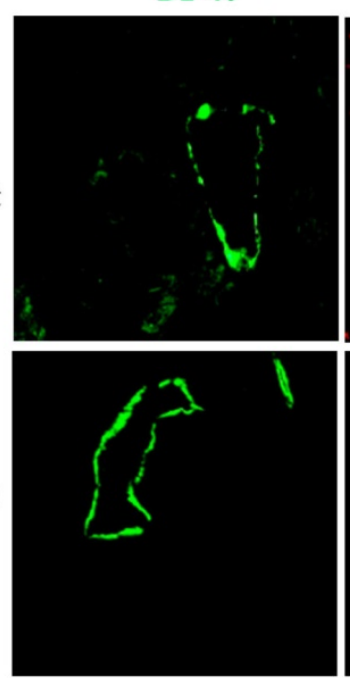

CD137
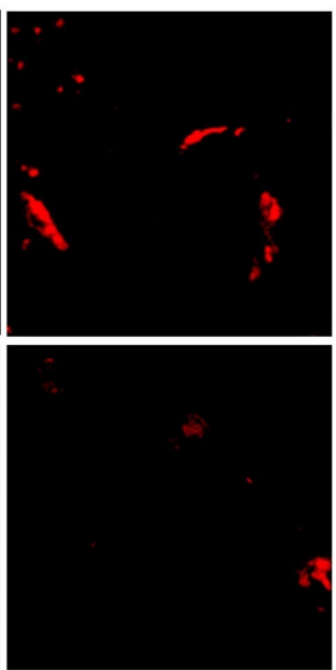

Merge
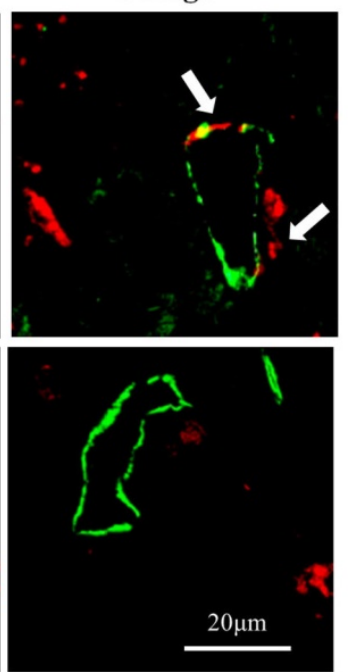

C
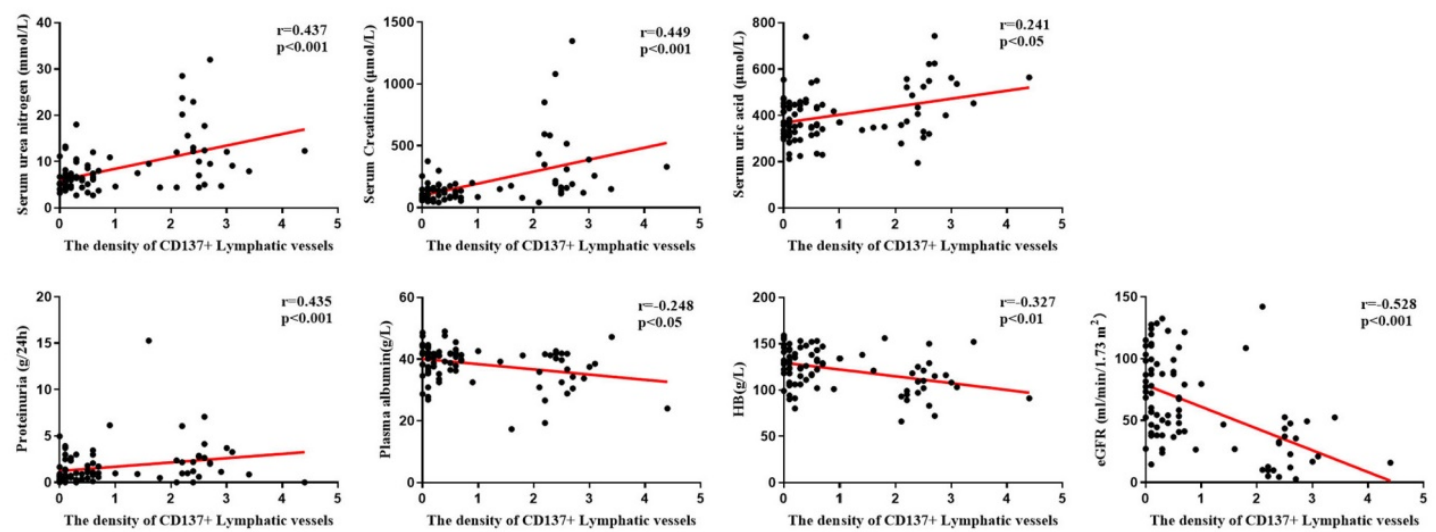

D
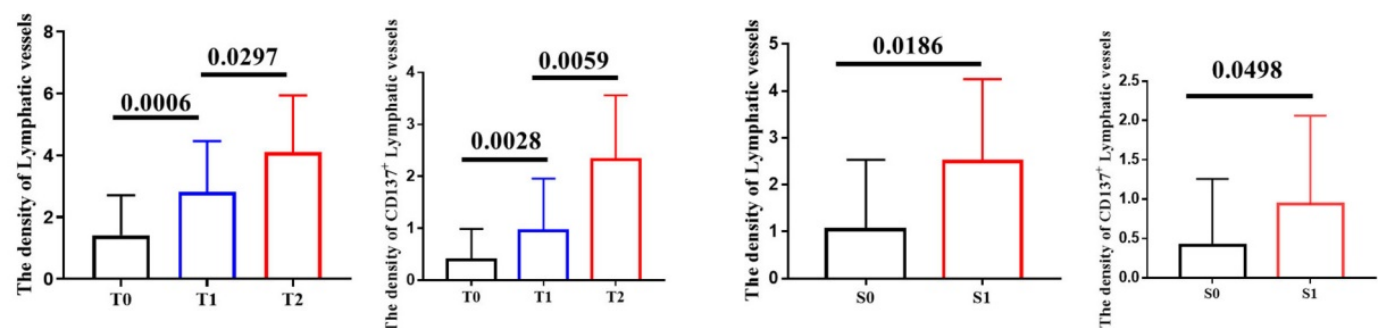

Figure 1. CD137 expression correlated with intrarenal lymphangiogenesis and fibrosis in IgAN. (A) IHC analysis of CD137 expression (40x); Masson's trichome staining showing collagen deposition(40x); (B) Representative images and double-positive analyses for immunofluorescence-labeled D2-40 (green) and CD137 (red) in IgA patients $(n=85)$ and controls $(n=5)$. Scale bar, 20 $\mu$ m. (C) Relationships between CD 137+ lymphatic vessels in the interstitium and Serum urea nitrogen, Serum Creatinine, Serum uric acid, proteinuria, Plasma albumin, HB and eGFR. HB, hemoglobin; eGFR, estimated glomerular filtration rate; (D) The difference of density of lymphatic vessels and CD137+lymphatic vessels between patients with different $\mathrm{T}$ and $\mathrm{S}$ grades. T, tubular atrophy/interstitial fibrosis; S, segmental glomerulosclerosis. 
Table 2. Demographic and clinical parameters between patients with low or high levels of CD137+D2-40 expression.

\begin{tabular}{|c|c|c|c|c|}
\hline Variables & $\operatorname{ALL}(n=85)$ & $\begin{array}{l}\text { CD137+D2-40 low } \\
(\mathrm{n}=61)\end{array}$ & $\begin{array}{l}\text { CD137+D2-40high } \\
(n=24)\end{array}$ & $p$ \\
\hline $\begin{array}{l}\text { Male gender } \\
(\%)\end{array}$ & $42.0(49.4 \%)$ & $31(50.8 \%)$ & $11(45.8 \%)$ & ns \\
\hline Age (years) & $34.0(28.0-44.0)$ & $34.0(28.5-45.0)$ & $33.5(27.0-37.5)$ & ns \\
\hline $\begin{array}{l}\text { Serum } \\
\text { Creatinine } \\
(\mu \mathrm{mol} / \mathrm{L})\end{array}$ & $119.0(83.0-189.0)$ & $98.0(76.5-148.0)$ & $\begin{array}{l}237.5 \\
(139.8-496.3)\end{array}$ & $<0.001$ \\
\hline $\begin{array}{l}\text { Serum urea } \\
\text { nitrogen } \\
(\mathrm{mmol} / \mathrm{L})\end{array}$ & 6.64(4.65-9.95) & $6.1(4.4-7.4)$ & $\begin{array}{l}12.05 \\
(5.52-17.18)\end{array}$ & $<0.001$ \\
\hline $\begin{array}{l}\text { Serum uric acid } \\
(\mu \mathrm{mol} / \mathrm{L})\end{array}$ & $375.0(325.0-454.0)$ & $359.0(318.9-437.5)$ & $\begin{array}{l}449.0 \\
(361.9-555.0)\end{array}$ & 0.0022 \\
\hline $\begin{array}{l}\text { eGFR } \\
(\mathrm{ml} / \mathrm{min} / 1.73 \\
\mathrm{m} 2)\end{array}$ & $54.2(36.15-90.25)$ & $72.1(46.7-101.4)$ & $\begin{array}{l}27.2 \\
(10.68-48.98)\end{array}$ & $<0.001$ \\
\hline $\begin{array}{l}\text { Proteinuria } \\
(\mathrm{g} / 24 \mathrm{~h})\end{array}$ & $1.0(0.59-2.49)$ & $0.51(0.29-1.06)$ & $1.0(0.74-2.29)$ & 0.0006 \\
\hline $\begin{array}{l}\text { Plasma albumin } \\
(\mathrm{g} / \mathrm{L})\end{array}$ & $40.1(35.8-41.95)$ & $40.8(36.83-42.35)$ & $38.0(31.3-41.75)$ & 0.0294 \\
\hline $\mathrm{HB}(\mathrm{g} / \mathrm{dL})$ & $123.5(106.25-142.5)$ & $129.0(116.25-144.00)$ & $108.5(93.5-124.0)$ & 0.0001 \\
\hline
\end{tabular}

Table 3. Histopathologic features from renal biopsies between patients with low or high levels of CD137+D2-40 expression.

\begin{tabular}{|c|c|c|c|c|}
\hline Variables & $\begin{array}{l}\operatorname{ALL}(n=74 \\
)\end{array}$ & $\begin{array}{l}\text { CD137+D2-40 } \\
\text { w }(n=55)\end{array}$ & $\begin{array}{l}\text { CD137 }{ }^{+} D 2-40^{\text {hig }} \\
\text { h }(n=19)\end{array}$ & $p$ \\
\hline \multicolumn{5}{|c|}{ Mesangial hypercellularity } \\
\hline M0 & $60(81.1 \%)$ & $43(78.2 \%)$ & $17(89.5 \%)$ & ns \\
\hline M1 & $14(18.9)$ & $12(21.8 \%)$ & $2(10.5 \%)$ & \\
\hline \multicolumn{5}{|c|}{$\begin{array}{l}\text { Segmental } \\
\text { glomerulosclerosis }\end{array}$} \\
\hline So & $7(9.5 \%)$ & $6(10.9 \%)$ & $1(5.3 \%)$ & ns \\
\hline S1 & $67(90.5 \%)$ & $49(89.1 \%)$ & $18(94.7 \%)$ & \\
\hline \multicolumn{5}{|c|}{$\begin{array}{l}\text { Endocapillary } \\
\text { hypercellularity }\end{array}$} \\
\hline E0 & $6(8.1 \%)$ & $4(7.3 \%)$ & $2(10.5 \%)$ & ns \\
\hline E1 & $68(91.9 \%)$ & $51(92.7 \%)$ & $17(89.5 \%)$ & \\
\hline \multicolumn{5}{|c|}{ Tubular intersititial fibrosis } \\
\hline T0 & $40(54.1 \%)$ & $37(67.3 \%)$ & $3(15.8 \%)$ & $<0.00$ \\
\hline $\mathrm{T} 1$ & $21(28.3 \%)$ & $15(27.3 \%)$ & $6(31.6 \%)$ & 1 \\
\hline $\mathrm{T} 2$ & $13(17.6 \%)$ & $3(5.5 . \%)$ & $10(52.6 \%)$ & \\
\hline \multicolumn{5}{|l|}{ Crescents } \\
\hline $\mathrm{CO}$ & $42(56.8 \%)$ & $32(58.2 \%)$ & $10(52.6 \%)$ & ns \\
\hline $\mathrm{C} 1$ & $28(37.8 \%)$ & $20(36.4 \%)$ & $8(42.1 \%)$ & \\
\hline $\mathrm{C} 2$ & $4(5.4 \%)$ & $3(5.5 \%)$ & $1(5.3 \%)$ & \\
\hline
\end{tabular}

Data are presented as percentages.

Pathologic features were scored using the Oxford classification.

\section{Renal CD137+ lymphatic vessels are increased in the UUO model}

To ascertain that damaged kidneys contain abundant $\mathrm{CD} 137^{+}$lymphatic vessels, we investigated the expression and location of LYVE-1 and CD137 in a UUO mouse model. Extensive stromal fibrosis was detected by Masson's trichrome staining, and increased CD137 and LYVE-1 expression and infiltrating macrophages were detected by immunohistochemistry (IHC) in mouse kidneys after the operation compared with those of the sham-operated mice; these parameters peaked on day 14 (Figures 2A). Western blotting and real-time PCR results indicated that the expression of lymphangiogenesis markers (Prox-1 and LYVE-1) and CD137 was significantly elevated in the kidneys of
UUO mice compared with sham-operated mice, which was consistent with the histopathological results. (Figure 2B, C). Meanwhile, we also observed that some LYVE-1+ ${ }^{+}$lymphatic vessels were also positive for CD137 in UUO mice (Figure 2D).

\section{CD137L induced by macrophages in the Kidney Following Obstructive Injury}

CD137-CD137L interactions have been implicated in acute kidney inflammation, and we proceeded to investigate the role of their interactions during the development of chronic kidney disease. As shown in Figure 3A, renal mRNA levels of CD137L were substantially increased in UUO mice compared with sham control mice. In addition, the serum level of CD137L in UUO mice was significantly increased in the early and late stages compared with that in the serum of sham control mice (Figure 3B). To further define which cells were the major source of CD137L production in UUO kidneys, we performed flow cytometry. The results showed that in the UUO groups, the percentages of $\mathrm{CD}^{2} 5^{+} \mathrm{CD} 11 \mathrm{~b}+\mathrm{F} 4 /$ $80^{+} \mathrm{CD} 137 \mathrm{~L}^{+}$macrophages were higher than those of the sham-operated group and increased with the extension of ligation (Figure 3C). Our previous investigation verified that lymphangiogenesis in renal fibrosis arises from macrophages, so we paid more attention to the relationship between macrophages and lymphangiogenesis. Consistent with the FACS data, immunofluorescence staining showed that some macrophages detected in the interstitium colocalized CD137L in UUO mice (Figure 3E).

\section{VEGF-C stimulates CD137L Expression on macrophages}

We firstly assessed the role of VEGF-C on macrophages by real-time PCR. As shown in Figure $4 \mathrm{~A}$, the expression of CD137L was increased in BMDMs treated with VEGF-C (50, 100, $1000 \mathrm{ng} / \mathrm{mL})$. The optimal VEGF-C concentration $(100 \mathrm{ng} / \mathrm{mL})$ was used for subsequent experiments. Multiple studies have confirmed that proinflammatory cytokines are abundantly expressed in the fibrosis model. To determine which cytokines induced the expression of CD137L on the surface of macrophages, we used TGF- $\beta$, IFN- $\gamma$, LPS, and VEGF-C to conduct cell stimulation experiments on peritoneal macrophages (MPMs) and bone marrow-derived macrophages (BMDMs) in vitro (Figure 4B-E). Flow cytometry results showed that only VEGF-C was able to induce the expression of CD137L on both MPMs and BMDMs, and other cytokines had no obvious effect on the expression of MPMs CD137L. Moreover, treatment of macrophages with VEGFR3 inhibitor SAR131675 partially decreased the expression of 
CD137L induced by VEGF-C. Considering many proinflammatory factors exist simultaneously in vivo, we used a mixture of proinflammatory cytokines to stimulate macrophages. The results showed that the expression of CD137L on macrophages increased sharply induced by VEGF-C, TGF- $\beta$ and IL-1 $\beta$ (Figure $4 F)$. Our data indicated that VEGF has synergism with other inflammatory factors on macrophages during inflammatory conditions in vivo. And we also confirmed the increased renal VEGV-C expression in UUO mice (Supplement Figures 2).

A
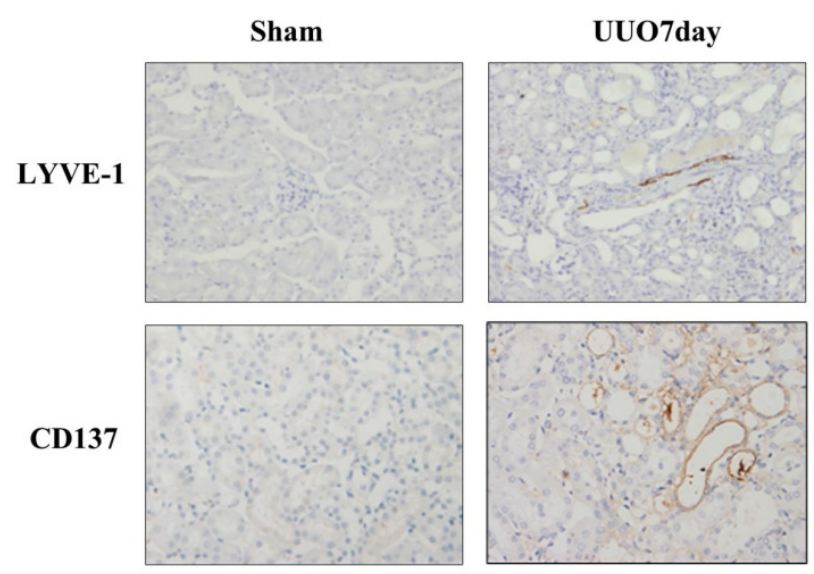

B

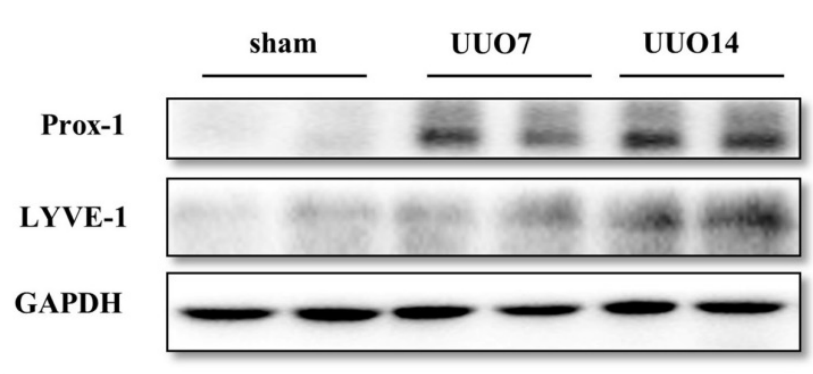

D
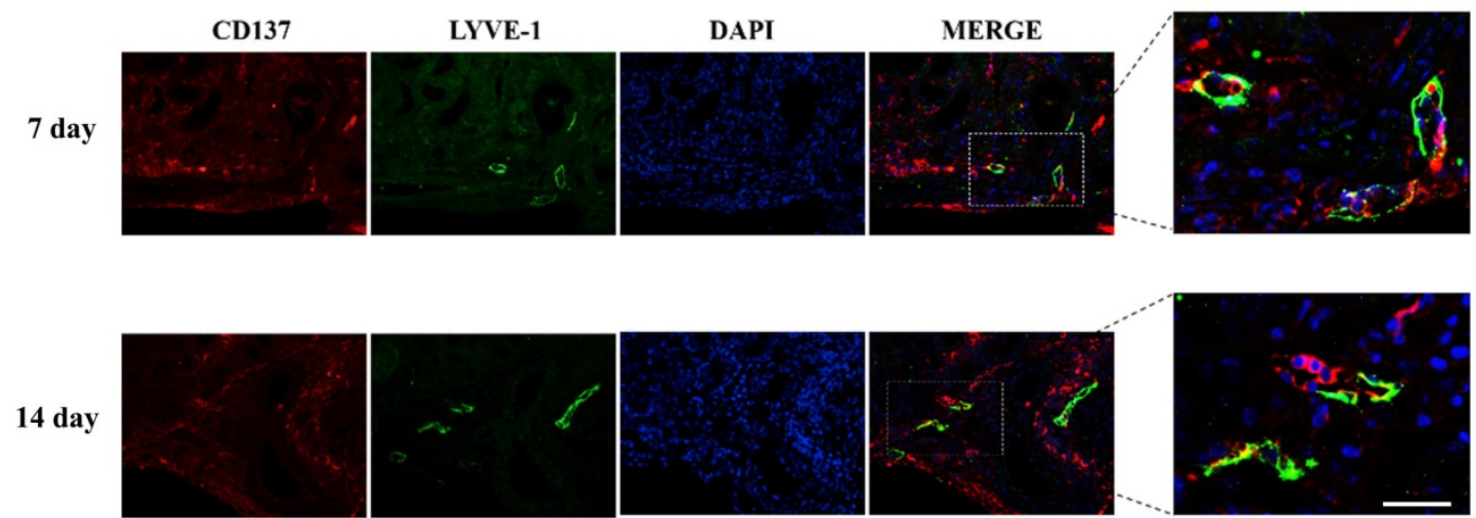

Figure 2. CD137 is increased in the obstructed kidney. (A) IHC staining showing LYVE-1 and CD137 expression in the sham control and UUO mice (400x). The expression levels of Prox-1 and LYVE-1 were detected by Western blot (B), and the expression levels of CD137 were detected by real-time PCR (C). (D) Immunofluorescence staining showing CD137 (red) expression in UUO kidneys in the interstitium on days 7 and 14 and colocalization with LYVE-1 (green) original magnification, $\times 400$. $n=6$ per group. The error bars represent the SEM. **** $<0.001$. 
A

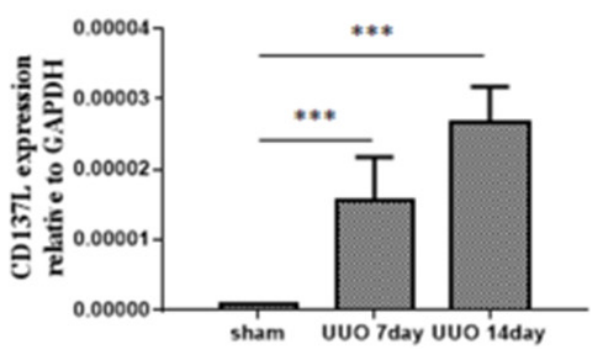

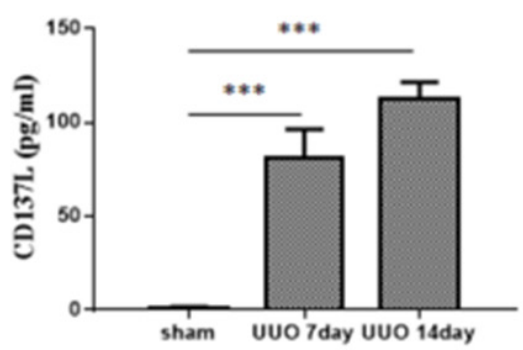

C

Scheme of cell sorting

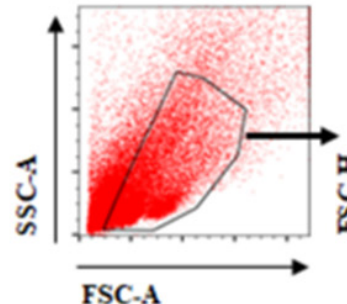

ESC-A

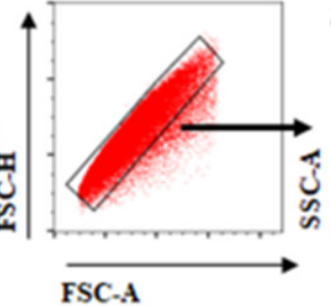

FSC-A

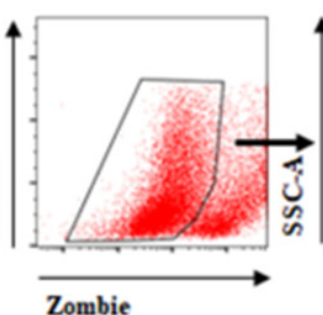

Zombie

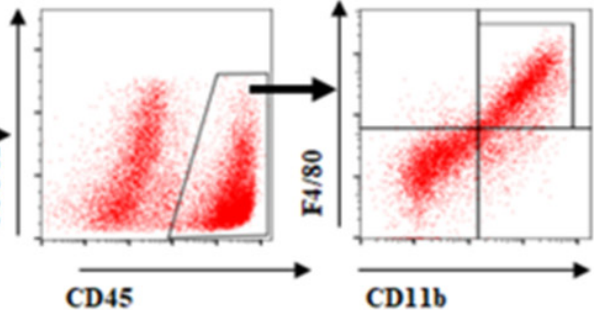

CD137L'Mo

(among $\mathrm{CD}^{+} 5^{+}$cells)

D

Normal

uto7d

Utol4d
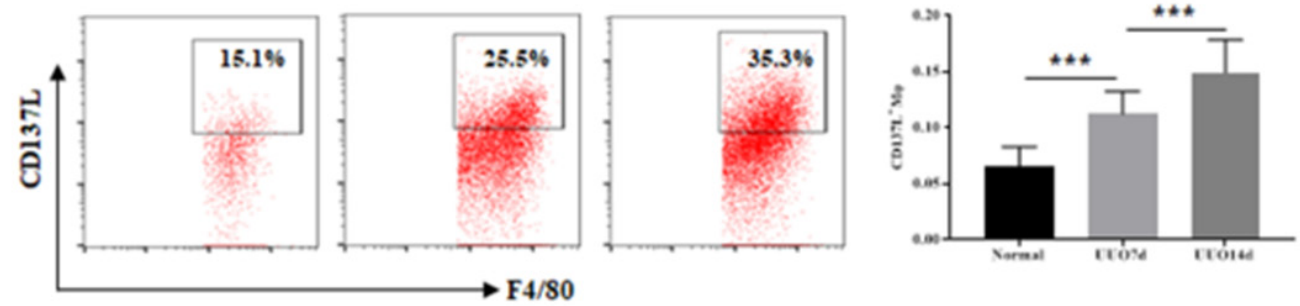

E

F4/80

CD137L

DAPI

Merge
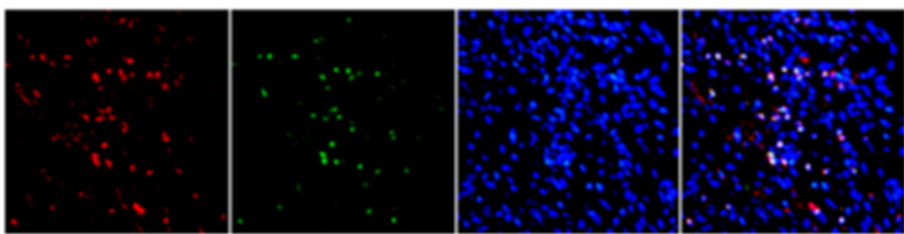

tUO 14d
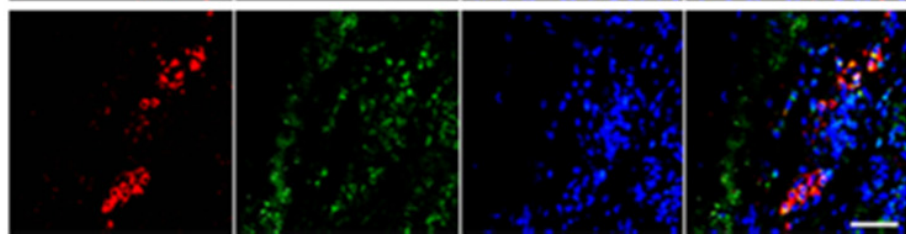

Figure 3. Expression of CD137L on macrophages in UUO mice. Mice were subjected to unilateral ureteral obstruction (UUO) by left ureteral ligation for 7 and 14 days. CD137L mRNA and protein expressions were detected by Real-time PCR (A) and Elisa (B). (C) Scheme of the cell-sorting approach. (D) Flow cytometric analysis and quantification of CD137L + macrophages in sham and UUO mice. kidney cell suspensions from the obstructed kidneys. (E) Immunofluorescence staining showing CD137L (green) expression and colocalization (white arrows) with F4/80 (red) in UUO kidneys. The error bars represent the SEM. ***P $<0.001$. Original magnification, Scale bar, $20 \mu \mathrm{m}$. $\mathrm{n}=6 \mathrm{per}$ group. The data were pooled from three independent experiments.

\section{The biological effects of CD137L on LECs in vitro}

Given that both CD137 on LECs and CD137L expression are elevated in the kidneys of UUO mice, we then proceeded to investigate the biological effects of CD137L on LECs in vitro. As shown in Figure 5A, increased cell growth was observed in SVEC4-10 cells after $48 \mathrm{~h}$ of culture in CD137L-containing media. The population-doubling time of SVEC4-10 cells with CD137L was significantly shorter than that of the control cell lines. The scratch test showed that CD137L on LECs for $12 \mathrm{~h}$ significantly increased the migration distance of LECs compared with that of the control group (Figure 5B). The Transwell chamber results showed that the number of migrating LECs upon 
culturing for $24 \mathrm{~h}$ with the addition of CD137L significantly increased 2- to 3-fold (Figure 5D, P < 0.001 ). Subsequently, the tube formation assay of LECs showed that CD137L also increased tube formation (Figure 5E). Moreover, treatment of LECs with CD137 inhibitory antibody partially inhibited the proliferation and decreased the migration distance and the number of migrating cells to the lower chamber and tube formation induced by CD137L. These findings indicate that the biological effects of
CD137L on LECs are largely dependent on the expression of its receptor, CD137.

\section{Transcriptome analysis of LECs after CD137L treatment}

It is clear from the above results that the CD137/CD137L network promotes LECs to exert its multiple functions. To further characterize the mechanism of LECs after CD137L treatment, we performed RNA sequencing (RNA-seq) on SVEC4-10

$\mathbf{A}$

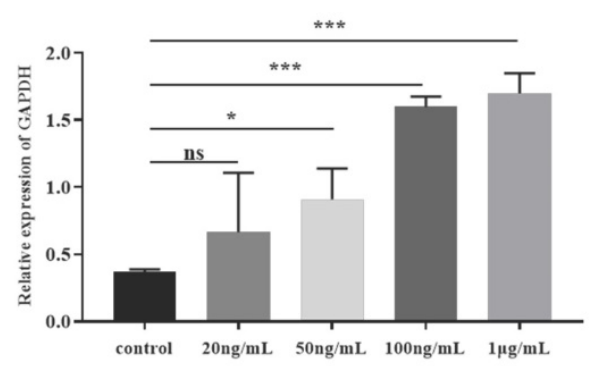

B
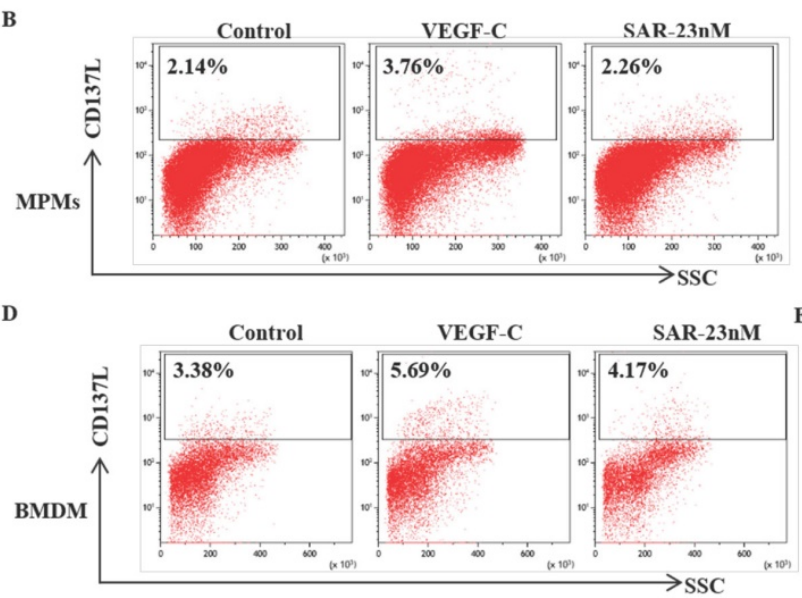

$\mathbf{F}$
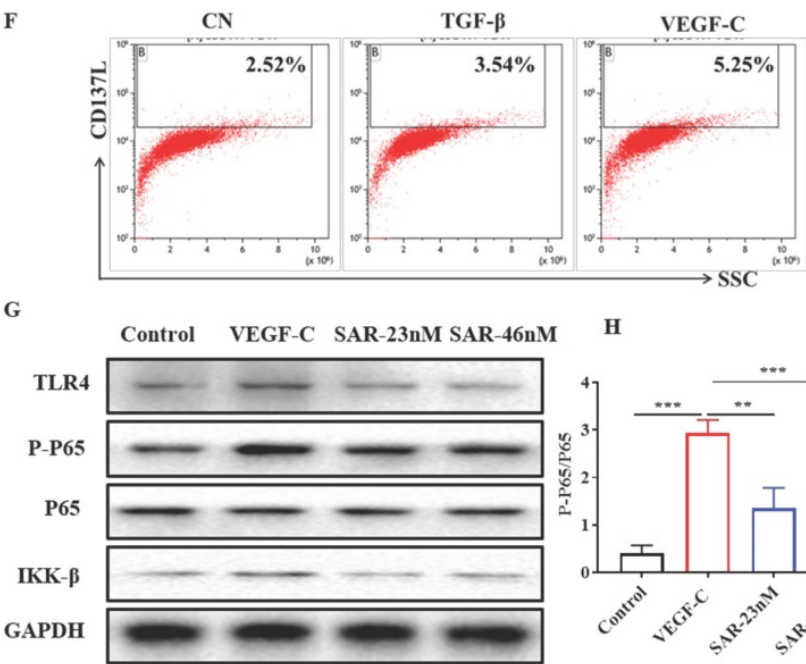

H
$\mathrm{C}$

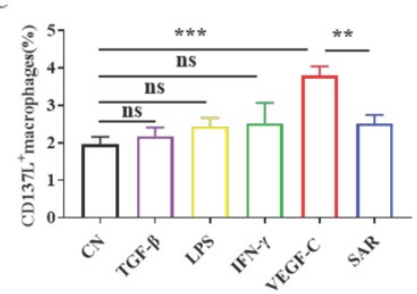

$\mathbf{E}$

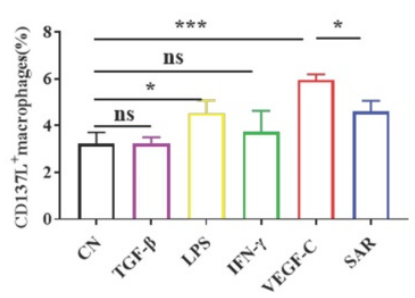

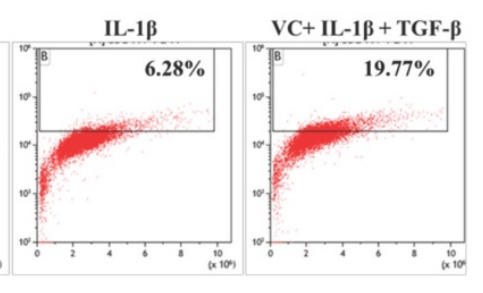
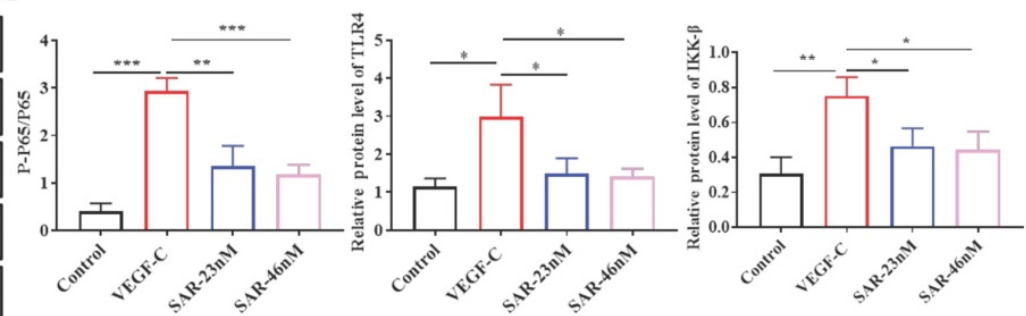

Figure 4. VEGF-C promotes CD137L production in macrophages in vitro. (A)Relative mRNA expression of CD137L was measured in BMDMs treated with VEGF-C (0, 20,50, $100,1000 \mathrm{ng} / \mathrm{mL}$ ) subpopulations by real-time PCR. Flow cytometric analysis and quantification showing the percentages of CDI37L+ MPMs (B, C) and BMDMs (D, E) with VEGF-C $(100 \mathrm{ng} / \mathrm{mL})$ and SAR131675 $(23 \mathrm{nM})$. The data shown were representative FACS profiles. (F) Flow cytometric analysis showing the percentages of CDI37L+BMDMs with TGF- $\beta(10 \mathrm{ng} / \mathrm{mL}), V E G F-C(100 \mathrm{ng} / \mathrm{mL}), \mathrm{L}-1 \beta(25 \mathrm{ng} / \mathrm{mL})$ and mixture of cytokines. The data shown were representative FACS profiles. (G) BMDMs were grown in complete medium and treated with VEGF-C $(100 \mathrm{ng} / \mathrm{mL})$ with or without SAR131675 $(23 \mathrm{nM}, 46 \mathrm{nM})$ for $48 \mathrm{~h}$. Western blot analysis was performed to measure the expression of TLR4, $\mathrm{P}-\mathrm{P} 65, \mathrm{P} 65$, IKK $-\beta$ and GAPDH. (H) The histograms show the relative intensity for each marker normalized to GAPDH. The error bars represent the SEM. *P< 0.05 , **P $<0.01$; $* * * \mathrm{P}<0.001$ versus control. The data were pooled from three independent experiments. 
cells to analyze their whole transcriptomic profiles. Kyoto Encyclopedia of Genes (Figure 6A) and Genomes (KEGG) analysis (Figure 6A) showed that one of the most prominent pathways in LECs upon CD137L treatment was the PI3K-Akt signaling pathway. Our results showed that CD137L remarkably decreased the phosphorylation levels of PI3K, Akt, and mTOR. GO enrichment analysis indicated that cell adhesion progress, cell migration progress and angiogenesis progress were activated in LECs after CD137L treatment (Figure 6C). Our data showed that CD137L treatment accelerated LEC migration and tube formation. However, the capacity of recombinant CD137L treatment was obviously suppressed by CD137 inhibitory antibody and autophagy inhibitor 3-MA.

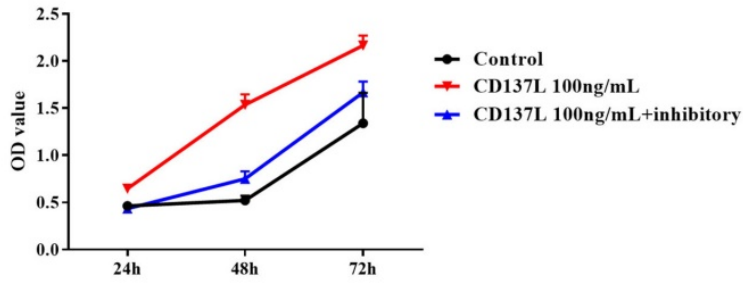

B

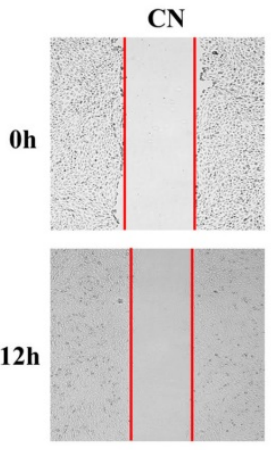

D

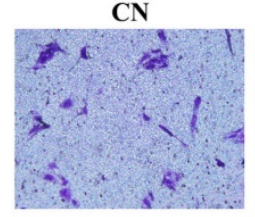

CD137L

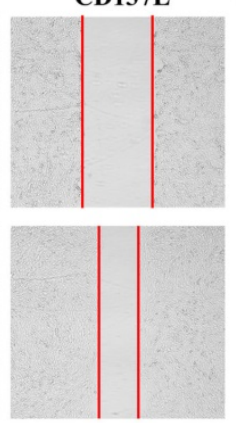

CD137L

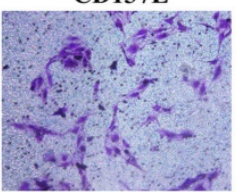

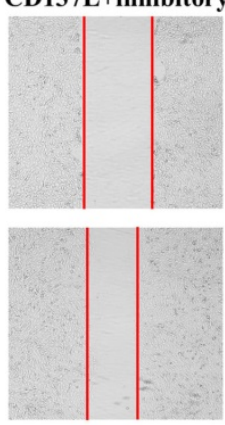

CD137L+inhibitory

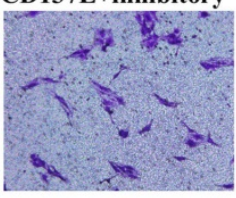

C

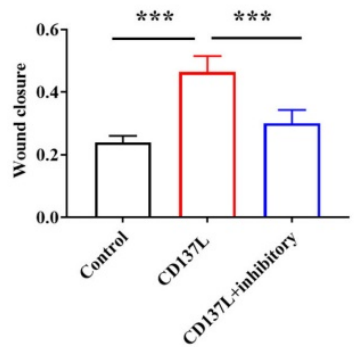

E

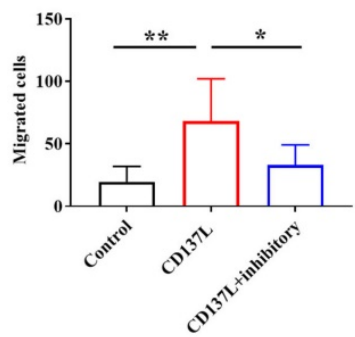

F

CN

CD137L
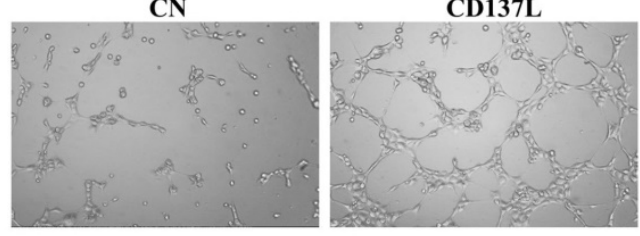

H

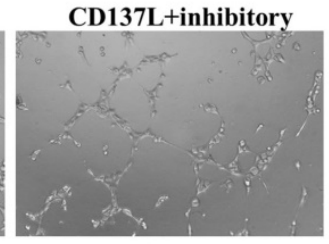

G
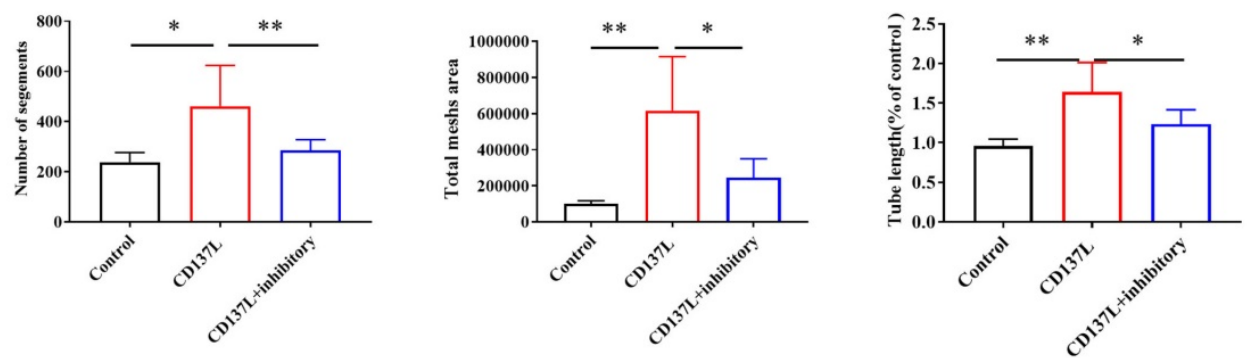

Figure 5. CD137L promotes the proliferation, migration and tube formation of lymphatic endothelial cells. (A) The proliferation of LECs treated with CD137L or CD137L and inhibitory for $24 \mathrm{~h}, 48 \mathrm{~h}$, and $72 \mathrm{~h}$ was examined by CCK-8 assay. The migration of LECs was measured with the cell scratch test (B) and Transwell migration assay (D) after CD137L or CD 137L and inhibitory treatment for $12 \mathrm{~h}$. The histograms represent migrated (C, E) cells per field. (F) A tube formation assay was used to test the lymphangiogenesis capacity of LECs after CD137L or CD137L and inhibitory treatment for $3 \mathrm{~h}$ (image magnification, 100x). The histograms represent the branch number per field. (G, H, I). The error bars represent the SEM. *P< 0.05 , **P $<0.01$; *** $\mathrm{P}<0.001$. The data were pooled from three independent experiments. 
A

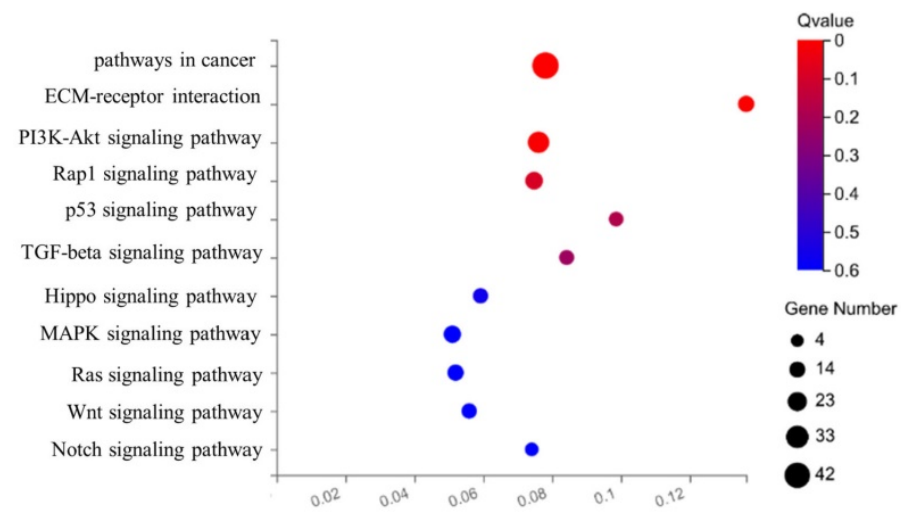

B

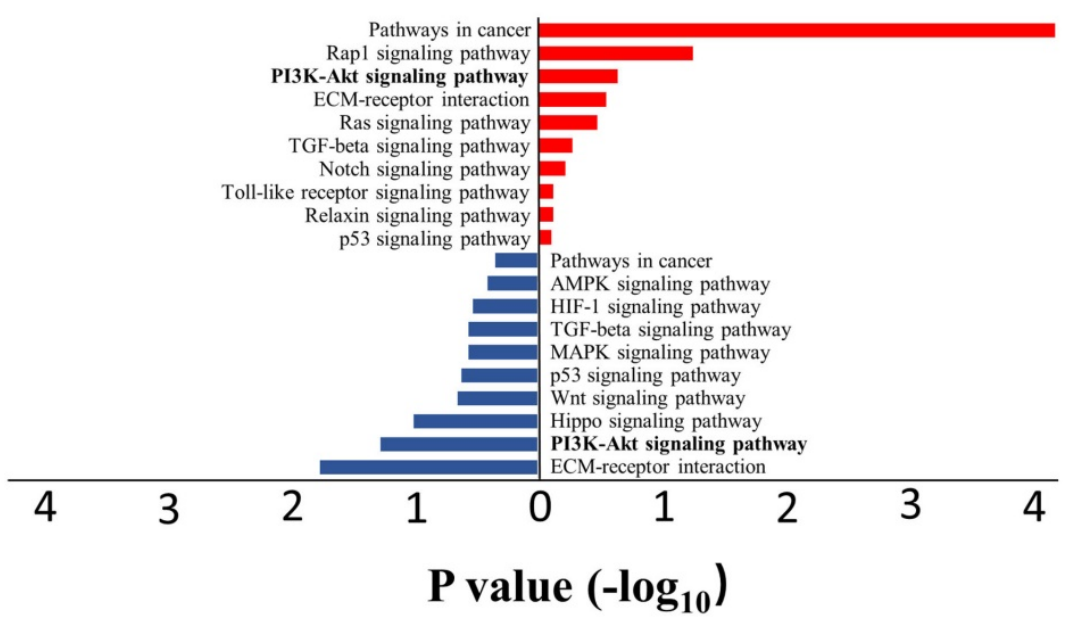

C

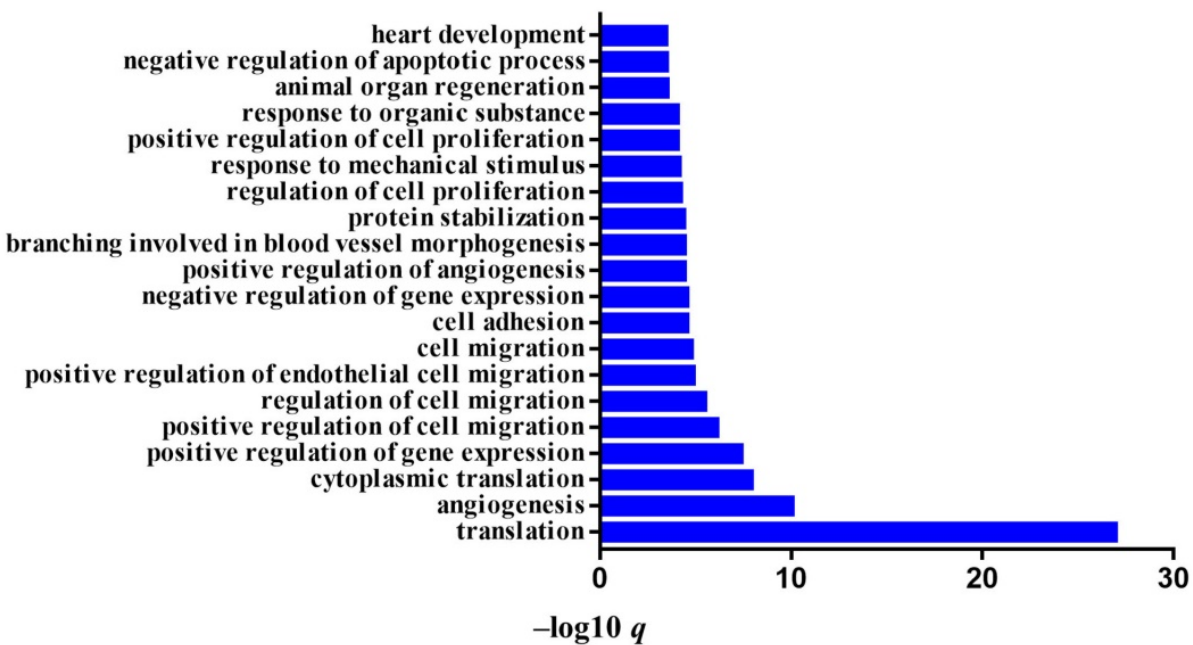

Figure 6. Transcriptome analysis of LECs after CD137L treatment (A) Eleven significantly activated KEGG pathway in SVEC4-10 cell line after treatment with CD137L. (B) Enriched KEGG pathway for differentially expressed genes in SVEC4-10 cells with or without CD137L treatment. The terms enriched from upregulated genes in CD137L treated SVEC4-10 cells are marked by red, and terms enriched from down regulated genes are marked by blue. (FDR $\leq 0.01)(C)$ Top20 significantly activated biological processes in SVEC4-10 cell line after treatment with CD137L, as indicated in GO analysis (FDR $\leq 0.01$ ). GO, gene ontology; DEGs, differentially expressed genes; FDR, false discovery rate; KEGG, Kyoto Encyclopedia of Genes and Genomes.

\section{CD137L induced the autophagy pathway via inactivation of the PI3K/AKT/mTOR signaling pathway}

Autophagy is initiated as a protective response to environmental stressors, and the excessive activation of autophagy can lead to the pathogenesis of cancer, cardiovascular and kidney disease. To demonstrate whether autophagy is involved in renal fibrosis, SVEC4-10 cells were treated with CD137L or CD137L plus inhibition, and the ultrastructure of the cells was analyzed by electron microscopy. The 
number of autophagic vesicles was also significantly increased in SVEC4-10 cells treated with CD137L for $24 \mathrm{~h}$, and the number of autophagic vesicles was reduced in cells treated with CD137L and inhibited for $24 \mathrm{~h}$ (Figure 7A). Moreover, we measured the expression of the autophagy-related proteins LC3II, P62, Atg5, Atg7 and Atg12 in LECs treated with CD137L. The expression levels of LC3II, Atg5, Atg7, and Atg12 were increased, and P62 expression was decreased. Cotreatment of LECs with inhibitory or 3-MA inhibited CD137L-induced autophagy (Figure 7B). Autophagy is a dynamic process and we try to clarify the effects CD137L exerts on LECs. We transfected SVEC4-10 cells using GFP-RFP-LC3 lentiviral particles and observed under confocal microscopy view. The autolysosomes were labeled with red dots, and the autophagosomes were both labeled with red dots and green dots. After incubating with recombinant $\mathrm{CD} 137 \mathrm{~L}$ protein for $24 \mathrm{~h}$, the number of autophagosomes (yellow dots, a merge of red dots and green dots) and autolysosomes (green dots) increased remarkably, while the number of both autophagosomes and autolysosomes did not increase significantly after pretreated with CD137 inhibitory. The process of autophagy was inhibited after treating with CD137L and 3-MA (10 mM) (Figure 7C). Moreover, the inhibition of CD137L-induced autophagy of LECs with 3-MA led to inhibited proliferation and decreased migration distance and the number of migrating cells to the lower chamber and tube formation induced by CD137L (Figure 7D, E and $\mathrm{F}$ ).

To investigate the mechanism by which CD137L induced autophagy, Western blotting was utilized to detect the PI3K/AKT/mTOR pathway. As shown in Figure 7G, treatment with CD137L decreased the levels of phosphorylated PI3K, Akt and mTOR in LECs. Cotreatment of LECs with inhibitory or 3-MA attenuated CD137L-induced signaling pathway inactivation. We next inhibited autophagy by Atg5 siRNA or Atg7 siRNA. The results showed that LECs transfected with siRNA specifically targeting Atg5 or Atg7 suppressed CD137L-induced autophagy, suggesting the requirement of these genes in CD137L-induced autophagy (Figure 7H).

\section{Discussion}

Our group previously reported that renal macrophage infiltration and lymphangiogenesis were strongly associated with the progression of fibrosis (7). In this study, we further analyzed the correlation between $\mathrm{CD} 137^{+}$lymphatic vessels and clinical parameters of $\operatorname{IgA}$ nephropathy patients and explored the influence of $\mathrm{CD} 137^{+}$lymphangiogenesis on the progression of IgA nephropathy to renal fibrosis.
Moreover, compared with lymphatic vessel densities, $\mathrm{CD}_{137^{+}}$lymphatic vessel densities were more associated with tubular atrophy/interstitial fibrosis. Then, we observed that CD137+ lymphatic vessels were involved in the development of $\operatorname{IgA}$ nephropathy and positively correlated with the severity of the disease. CD137 is mainly expressed in $\mathrm{T}$ lymphocytes and natural killer (NK) cells and in noninflammatory cells such as endothelial cells (24-26). Teijeira and colleagues have provided compelling evidence for inflammation-dependent expression of CD137 on the surface of LECs in vitro. CD137 ${ }^{+}$lymphatic vessels were found in the dermis of patients diagnosed with inflamed or infected skin ulcers, while they were lacking in the lymphatic vessels of noninflamed tissues (27). Our research also confirmed that CD137 was strikingly expressed in lymphatic endothelial cells in IgA patients. Similar results were confirmed in the UUO mice model.

During renal fibrosis, macrophages are actively involved in promoting lymphangiogenesis in two ways. One is to transdifferentiate into lymphatic vessels in human kidney transplants $(28,29)$, in a UUO mouse model (30), and in murine tumor models (31). Another way is to secrete prolymphangiogenic growth factors such as VEGF-C, VEGF-D, and VEGF-A in response to inflammatory mediators (32-35). In op/op mice, lymphatic vessel development is delayed due to the lack of macrophages, which demonstrates that macrophages seem to be important sources of lymphangiogenic factors (36). In this study, we found that only VEGF-C, but not inflammatory cytokines (IFN- $\gamma$ and LPS), could stimulate CD137L expression on both peritoneal macrophages (MPMs) and bone marrow-derived macrophages (BMDMs). This reason can be explained by the heterogeneity of macrophages. BMDMs, as circulating macrophages, are ex vivo differentiated from stem cells and are more responsive to environments. MPMs, as resident macrophages, are minimally replaced by blood monocytes under homeostatic conditions $(37,38)$. BMDM responses are a function of intrinsic differences, while MPM responses reflect their differentiation in the context of the whole animal. In previous studies, we also noticed a difference between resident and circulating macrophages. We found that the level of local C3 mostly secreted by resident macrophages was highly correlated with RF, while glomerular C3 deposition secreted by infiltrating macrophages was also associated with the severity of renal lesions (39). These results suggest that resident macrophages not only phagocytose the environment but also respond rapidly to the environment and release cytokines. 
A

CN
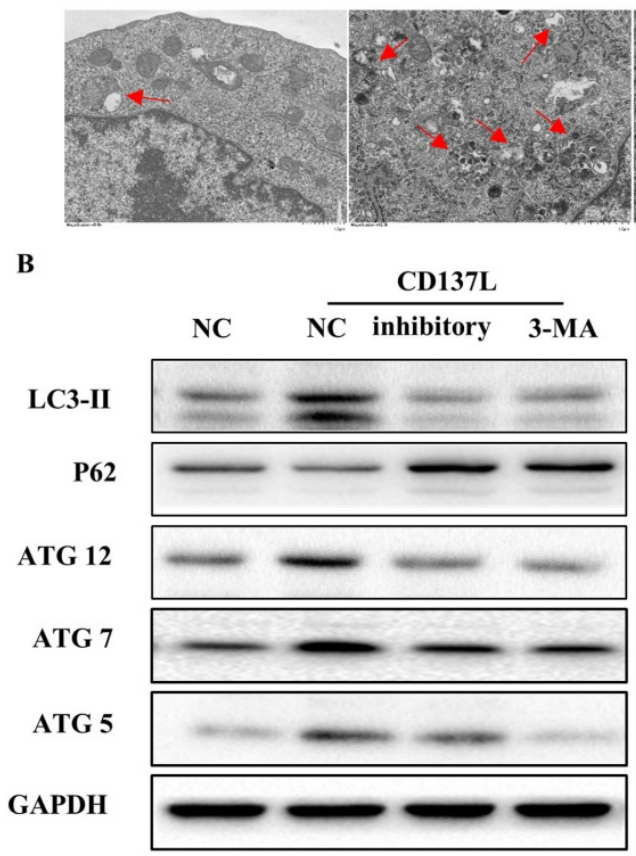

F

CD137L CD137L+3-MA

o h

$12 \mathrm{~h}$

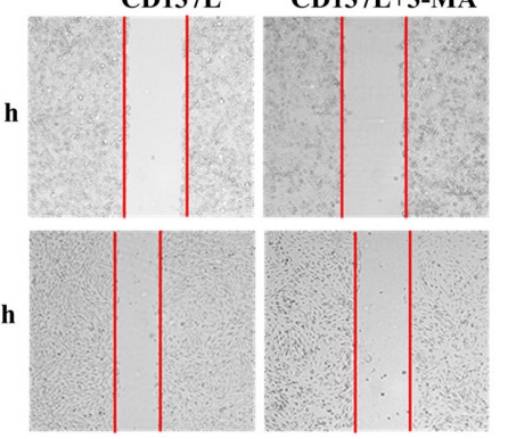

G

CD137L
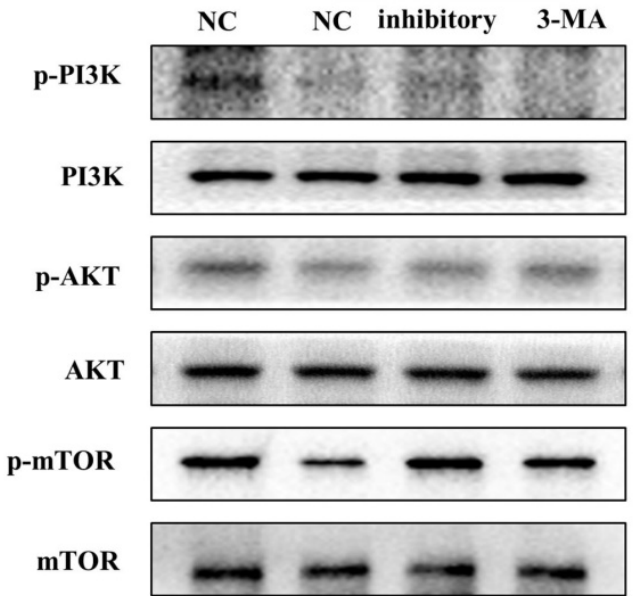

$\beta$-actin

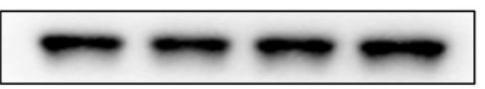

CD137L+inhibitory $\quad$ CD137L+3-MA
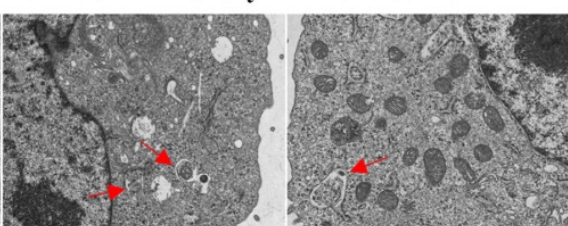

C
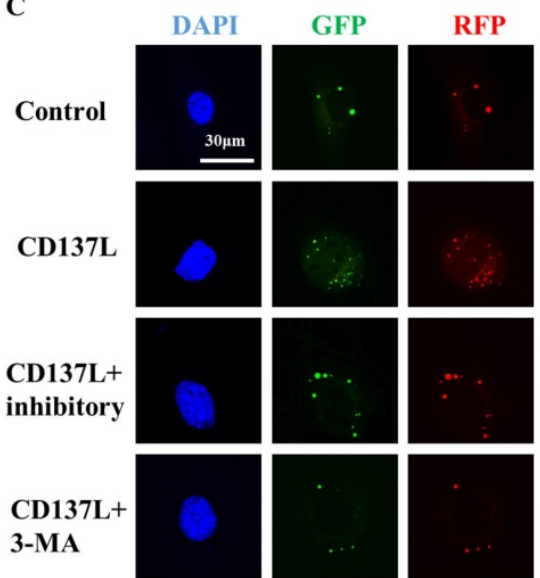

Merge
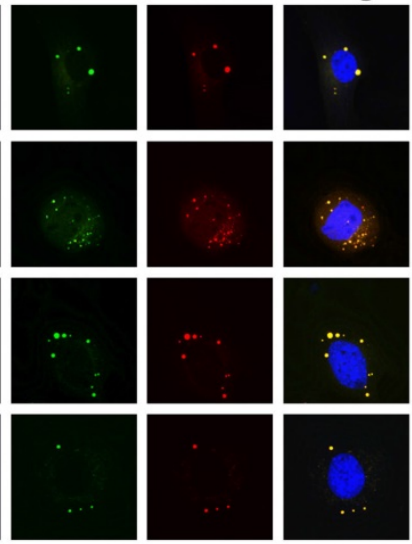

D

CD137L

CD137L+3-MA
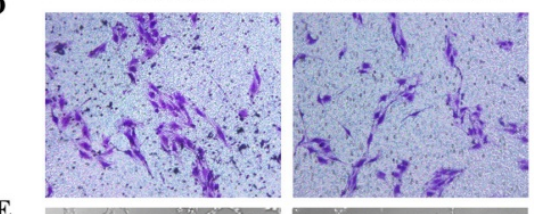

E
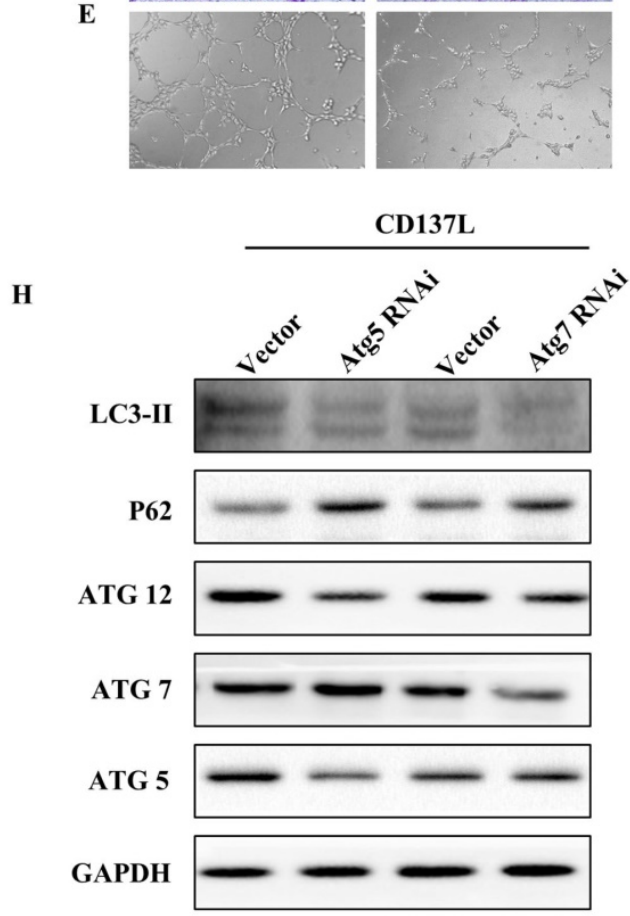

Figure 7. CD137L induced autophagy of lymphatic endothelial cells via mTOR pathway. (A) Representative electron micrographs of CD137L-induced autophagosome in SVEC4-10 cells that were either stimulated with inhibitory, or with 3-MA for $24 \mathrm{~h}$. (B) The protein expression levels of autophagy-associated proteins (Atg5, Atg7 and Atg12) and autophagic substrates (LC3 and p62) were analyzed in SVEC4-10 cells after CD137L, inhibitory or 3-MA treatment for 24 h by Western blot. (C) SVEC4-10 cells were infected with RFP-GFP-LC3-expressing lentivirus. Cells were untreated or treated with CD137L, inhibitory or 3-MA for 24 h. Fluorescence was examined by confocal microscopy. Transwell migration assay (D), scratch test (E) and tube formation assay (F) stimulated with CD137L for $12 \mathrm{~h}$, and with or without 3-MA treatment. (G) The protein expression levels of phosphorylated PI3K, Akt and mTOR were analyzed in SVEC4-10 cells after CD137L or inhibitory or 3-MA treatment for 24 h by Western blot. (H) SVEC410 cells were knocked down Atg5 and Atg7 by using siRNAs, and stimulated with CD137L for 24h, the protein expression levels of autophagy-associated proteins (Atg5, Atg7 and Atg12) and autophagic substrates (LC3 and p62) were detected by Western blotting. 


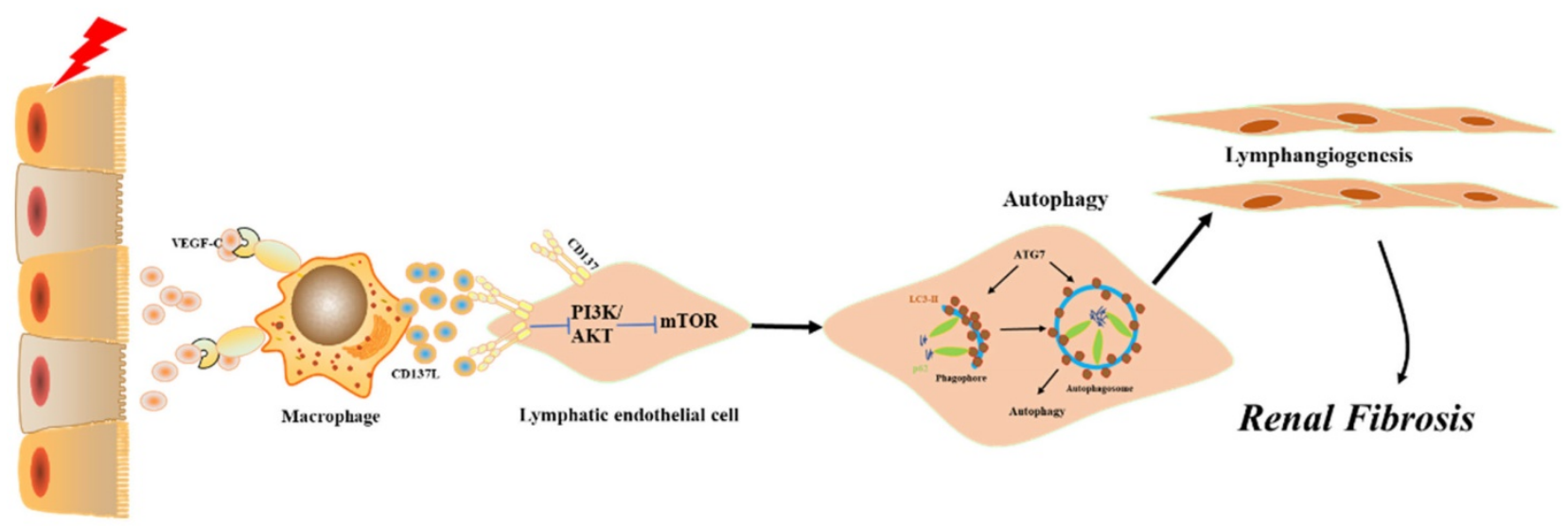

Figure 8. Model summarizing the role of $C D 137^{+}$lymphatic vessels participate in renal fibrosis. CDI37L secretion by macrophages leads to LEC autophagy, involving the inactivation of $\mathrm{mTOR}$ and $\mathrm{PI} 3 \mathrm{~K}$ signaling pathway.

VEGF-C is a classic lymphatic growth factor produced by circulating macrophages, podocytes and tubule epithelial cells (40-42). It promotes expansion and reconstruction of the lymphatic capillary net through activation of VEGFR-3 on LECs, leading to lymphangiogenesis in chronic kidney diseases. In acute kidney injury, pathogen-associated molecular patterns (PAMPs) and damage-associated molecular patterns (DAMPs) activate resident macrophages and kidney parenchymal cells to secrete inflammatory cytokines and chemokines $(43,44)$. Our research found that at the beginning of injury, damaged parenchymal cells released VEGF-C and directly activated resident macrophages through TLR4, which mediated rapid activation of NF- $\kappa \mathrm{B}$ and thereby stimulated the expression of CD137L. We also confirmed that VEGF-C could activate circulating macrophages to stimulate the expression of CD137L. Notably, resident and infiltrating macrophages may indirectly and directly participate in different phases of kidney disease by VEGF-C.

CD137-CD137L signaling participated in multiple stages of inflammation. CD137 is expressed on activated T cells, B cells, monocytes and NK cells. Recently, CD137 expression was also confirmed on the dermal lymphatic endothelial cells and blood endothelial cells of inflamed tissue samples, in which cross-linking with CD137L resulted in NF-kB nuclear translocation, followed by increased production of VCAM and CCL21 $(27,45-47)$. However, there are no reports regarding the expression and functionality of CD137 in renal lymphatic endothelial cells (LECs). In ischemic acute kidney injury, increased CD137 on infiltrating NK cells induces recruitment of neutrophils by stimulating the CD137L signaling pathway in TECs, which leads to their production of CXCL1 and CXCL2 through increased phosphorylation of p38 and JNK kinases and affects the degradation rate of IKBa (21). Similar to IRI, we found that CD137 was upregulated on lymphatic endothelial cells in IgA nephropathy patients, and the density of $\mathrm{CD} 137^{+}$lymphangiogenesis was positively correlated with renal interstitial fibrosis.

Autophagy dysfunction is involved in the pathogenesis of various renal diseases (48-51). In patients with $\mathrm{CKD}$, autophagy activities may increase to remove uremic toxin-induced injured components, which may have a protective role against CKD progression (52). However, in a mouse UUO model and TGF- $\beta 1$-treated PTECs, the effects of autophagy in renal interstitial fibrosis were controversial. Under this condition, protein kinase $\mathrm{C}$ (PKC)- $\alpha$, by stimulating autophagic flux, promoted autophagy and apoptosis in renal tubules and drove renal interstitial fibrosis (53). In contrast, other studies show that autophagy deficiency in proximal tubules leads to G2/M cell cycle arrest and increased production of collagen I, which accelerates renal interstitial fibrosis (54). Inhibition of autophagy by 3-methyladenine promoted fibrosis by inducing tubular cell apoptosis in a rat model of UUO $(55,56)$. The inconsistency of these results may be related to many types of renal resident cells, including podocytes, mesangial cells, glomerular endothelial cells, renal tubular epithelial cells and lymphatic endothelial cells. The role of autophagy in lymphatic endothelial cells is poorly understood. In this study, we found that the induction of autophagy by CD137L in lymphatic endothelial cells was mediated by the PI3K/AKT/mTOR signaling pathway. Enhanced autophagy led to increased proliferation, migration and tube formation of lymphatic endothelial cells, which ultimately formed lymphangiogenesis and promoted renal interstitial fibrosis.

Combined with the data obtained from patient samples, relevant animal experiments and cell models, our study defines a novel mechanism by which $\mathrm{CD} 137^{+}$lymphatic vessels participate in renal 
fibrosis. In response to injury, CD137L secretion by macrophages leads to LEC autophagy, which further drives lymphangiogenesis responses. Our present results add to the growing body of evidence supporting lymphangiogenesis as a potential therapeutic approach for obstructive nephropathy.

\section{Supplementary Material}

Supplementary figures and tables. https://www.ijbs.com/v18p1171s1.pdf

\section{Acknowledgments}

The authors thank all of our colleagues working in the Department of Nephrology, Tongji Hospital of Tongji Medical College, Huazhong University of Science and Technology.

\section{Author Contributions}

The experiments were conceived and designed by $\mathrm{YyL}, \mathrm{XjL}$ and $\mathrm{HtW}$. Experiments were performed by $\mathrm{HtW}, \mathrm{YqL}$ and LC. Data analyzed by KW, YZ, and QL. The paper was written by YyL and GX with input from all authors.

\section{Funding}

This work was partly supported by the National Natural Science Foundation of China (No. 81974088, 81772499 and 81800611).

\section{Competing Interests}

The authors have declared that no competing interest exists.

\section{References}

1. Wick G, Grundtman C, Mayerl C, Wimpissinger TF, et al. The immunology of fibrosis. Annu Rev Immunol. 2013; 31:107-35.

2. Graham-Brown MP, Singh A, Wormleighton J, et al. Association between native T1 mapping of the kidney and renal fibrosis in patients with IgA nephropathy. BMC Nephrol. 2019; 20(1):256

3. Lin $Q$, Chen $Y, \operatorname{Lv~J}$, et al. Kidney injury molecule-1 expression in IgA nephropathy and its correlation with hypoxia and tubulointerstitial inflammation. Am J Physiol Renal Physiol. 2014; 306(8): F885-95.

4. Imig JD, Ryan MJ. Immune and inflammatory role in renal disease. Compr Physiol. 2013; 3: 957-76.

5. Knauf F, Brewer JR, Flavell RA. Immunity, microbiota and kidney disease. Nat Rev Nephrol. 2019; 15(5):263-74.

6. Shi Y, Wang Y, Li Q, et al. Immunoregulatory mechanisms of mesenchymal stem and stromal cells in inflammatory diseases. Nat Rev Nephrol. 2018; 14(8):493-507.

7. Pei G, Zeng R, Han M, et al. Renal interstitial infiltration and tertiary lymphoid organ neogenesis in IgA nephropathy. Clin J Am Soc Nephrol. 2014; 9(2):255-64.

8. Tammela T, Alitalo K. Lymphangiogenesis: Molecular mechanisms and future promise. Cell. 2010; 140(4):460-76.

9. Weichand B, Popp R, Dziumbla S, et al. S1PR1 on tumor-associated macrophages promotes lymphangiogenesis and metastasis via NLRP3/IL-1 $\beta$ J Exp Med. 2017; 214(9):2695-713.

10. Kim H, Kataru RP, Koh GY. Inflammation-associated lymphangiogenesis: a double-edged sword? J Clin Invest. 2014; 124(3): 936-42.

11. Alitalo K, Tammela T, Petrova TV. Lymphangiogenesis in development and human disease. Nature. 2005; 438(7070): 946-53.

12. Ocansey DKW, Pei B, Xu X, et al. Cellular and molecular mediators of lymphangiogenesis in inflammatory bowel disease. J Transl Med. 2021; 19(1):254.

13. Gutierrez-Miranda L, Yaniv K. Cellular Origins of the Lymphatic Endothelium: Implications for Cancer Lymphangiogenesis. Front Physiol. 2020; $11: 577584$
14. Donnan MD, Kenig-Kozlovsky Y, Quaggin SE. The lymphatics in kidney health and disease. Nat Rev Nephrol. 2021; 17(10):655-75.

15. Sanchez-Paulete AR, Labiano S, Rodriguez-Ruiz ME, et al. Deciphering CD137 (4-1BB) signaling in T-cell costimulation for translation into successful cancer immunotherapy. Eur J Immunol. 2016; 46(3):513-22.

16. Melero I, Murillo O, Dubrot J, et al. Multi-layered action mechanisms of CD137 (4-1BB)-targeted immunotherapies. Trends Pharmacol Sci. 2008; 29(8):383-90.

17. Wang $\mathrm{C}$, Lin $\mathrm{GH}, \mathrm{McPherson} \mathrm{AJ}$, et al. Immune regulation by $4-1 \mathrm{BB}$ and 4-1BBL: complexities and challenges Immunol Rev. 2009; 229(1):192-215.

18. $\mathrm{Tu} \mathrm{TH}$, Kim $\mathrm{CS}$, Goto $\mathrm{T}$, et al. 4-1BB/4-1BBL interaction promotes obesity-induced adipose inflammation by triggering bidirectional inflammatory signaling in adipocytes/macrophages. Mediators Inflamm. 2012; 2012:972629.

19. Zeng Q, Zhou YB, Schwarz H. CD137L-DCs, Potent Immune-StimulatorsHistory, Characteristics, and Perspectives. Front Immunol. 2019; 10: 2216.

20. Zhang BY, Zhang YY, Niu LG, et al. Dendritic cells and Stat3 are essential for CD137-induced CD8 T cell activation-induced cell death. J Immunol. 2010; 184(9): 4770-8

21. Kim HJ, Lee JS, Kim JD, et al. Reverse signaling through the costimulatory ligand CD137L in epithelial cells is essential for natural killer cell-mediated acute tissue inflammation. Proc Natl Acad Sci USA. 2012; 109(1): E13-22.

22. Ho WT, Pang WL, Chong SM, et al. Expression of CD137 on Hodgkin and Reed-Sternberg cells inhibits T-cell activation by eliminating CD137 ligand expression. Cancer Res. 2013; 73(2): 652-61.

23. Trimarchi H, Barratt J, Cattran DC, et al. Oxford Classification of IgA nephropathy 2016: an update from the IgA Nephropathy Classification Working Group. Kidney Int. 2017; 91(5):1014-21.

24. Myers LM, Vella AT. Interfacing T-cell effector and regulatory function through CD137 (4-1BB) co-stimulation. Trends Immunol. 2005; 26(8):440-6.

25. Vidard L, Dureuil C, Baudhuin J, et al. CD137 (4-1BB) Engagement Fine-Tunes Synergistic IL-15- and IL-21-Driven NK Cell Proliferation. J Immunol. 2019; 203(3):676-85.

26. Xu L, Geng T, Zang G, et al. Exosome derived from CD137-modified endothelial cells regulates the Th17 responses in atherosclerosis. J Cell Mol Med. 2020; 24(8):4659-67.

27. Teijeira Á, Palazón A, Garasa S, et al. CD137 on inflamed lymphatic endothelial cells enhances CCL21-guided migration of dendritic cells. FASEB J. 2012; 26(8):3380-92.

28. Kerjaschki D, Regele HM, Moosberger I, et al. Lymphatic neoangiogenesis in human kidney transplants is associated with immunologically active lymphocytic infiltrates. J Am Soc Nephrol. 2004; 15(3):603-12.

29. Kerjaschki D, Huttary N, Raab I, et al. Lymphatic endothelial progenitor cells contribute to de novo lymphangiogenesis in human renal transplants. Nat Med. 2006; 12(2):230-4.

30. Zhang Y, Zhang C, Li L, et al. Lymphangiogenesis in renal fibrosis arises from macrophages via VEGF-C/VEGFR3-dependent autophagy and polarization. Cell Death Dis. 2021; 12(1):109.

31. Zumsteg A, Baeriswyl V, Imaizumi N, et al. Myeloid cells contribute to tumor lymphangiogenesis. PLoS One. 2009; 4(9): e7067.

32. Wang Y, Nakayama M, Pitulescu ME, et al. Ephrin-B2 controls VEGF-induced angiogenesis and lymphangiogenesis. Nature. 2010; 465(7297):483-6.

33. Li WN, Hsiao KY, Wang CA, et al. Extracellular vesicle-associated VEGF-C promotes lymphangiogenesis and immune cells infiltration in endometriosis. Proc Natl Acad Sci USA. 2020;117(41): 25859-68.

34. Cadamuro M, Brivio S, Mertens J, et al. Platelet-derived growth factor-D enables liver myofibroblasts to promote tumor lymphangiogenesis in cholangiocarcinoma. J Hepatol. 2019; 70(4): 700-9.

35. Tan KW, Chong SZ, Wong FHS, et al. Neutrophils contribute to inflammatory lymphangiogenesis by increasing VEGF-A bioavailability and secreting VEGF-D. Blood. 2013; 122(22): 3666-77.

36. Kubota Y, Takubo K, Shimizu T, et al. M-CSF inhibition selectively targets pathological angiogenesis and lymphangiogenesis. J Exp Med. 2009; 206(5):1089-102.

37. Zajd CM, Ziemba AM, Miralles GM, et al. Bone Marrow-Derived and Elicited Peritoneal Macrophages Are Not Created Equal: The Questions Asked Dictate the Cell Type Used. Front Immunol. 2020; 11:269.

38. Zimmerman KA, Yang ZQ, Lever JM, et al. Kidney resident macrophages in the rat have minimal turnover and replacement by blood monocytes. Am J Physiol Renal Physiol. 2021; 321(2): F162-9.

39. Liu $Y$, Wang K, Liang X, et al. Complement C3 Produced by Macrophages Promotes Renal Fibrosis via IL-17A Secretion. Front Immunol. 2018; 9:2385.

40. Kinashi $H$, Ito $Y$, Sun $T$, et al. Roles of the TGF- $\beta$-VEGF-C Pathway in Fibrosis-Related Lymphangiogenesis. Int J Mol Sci. 2018; 19(9):2487.

41. Foster RR, Slater SC, Seckley J, et al. Vascular endothelial growth factor-C, a potential paracrine regulator of glomerular permeability, increases glomerular endothelial cell monolayer integrity and intracellular calcium. Am J Pathol. 2008; 173(4): 938-48.

42. Chang $X$, Yang $Q$, Zhang $C$, et al. Roles for VEGF-C/NRP-2 axis in regulating renal tubular epithelial cell survival and autophagy during serum deprivation. Cell Biochem Funct. 2019; 37(4):290-300.

43. Rosin DL, Okusa MD. Dangers within: DAMP responses to damage and cell death in kidney disease. J Am Soc Nephrol. 2011; 22(3):416-25.

44. Cao Q, Harris DC, Wang Y. Macrophages in kidney injury, inflammation, and fibrosis. Physiology. 2015; 30(3): 183-94. 
45. Somekh I, Thian M, Medgyesi D, et al. CD137 deficiency causes immune dysregulation with predisposition to lymphomagenesis. Blood. 2019; 134(18): 1510-6.

46. Xu L, Geng T, Zang G, et al. Exosome derived from CD137-modified endothelial cells regulates the Th17 responses in atherosclerosis. J Cell Mol Med. 2020; 24(8):4659-67.

47. Yuan W, Xu C, Li B, et al. Contributions of Costimulatory Molecule CD137 in Endothelial Cells. J Am Heart Assoc. 2021; 10(11):e020721.

48. Zhao XC, Livingston MJ, Liang XL, et al. Cell Apoptosis and Autophagy in Renal Fibrosis. Adv Exp Med Biol. 2019; 1165:557-84

49. Ma Z, Li L, Livingston MJ, et al. p53/microRNA-214/ULK1 axis impairs renal tubular autophagy in diabetic kidney disease. J Clin Invest. 2020; 130(9):5011-26.

50. Yamamoto T, Takabatake $Y$, Minami S, et al. Eicosapentaenoic acid attenuates renal lipotoxicity by restoring autophagic flux. Autophagy. 2021; 17(7):1700-13.

51. Tang C, Han H, Yan M, et al. PINK1-PRKN/PARK2 pathway of mitophagy is activated to protect against renal ischemia-reperfusion injury. Autophagy. 2018; 14(5): 880-97.

52. Kim JK, Park MJ, Lee HW, et al. The relationship between autophagy, increased neutrophil extracellular traps formation and endothelial dysfunction in chronic kidney disease. Clin Immunol. 2018; 197:189-97.

53. Xue $X$, Ren J, Sun $X$, et al. Protein kinase $C$ a drives fibroblast activation and kidney fibrosis by stimulating autophagic flux. J Biol Chem. 2018; 293(28):11119-30.

54. Li H, Peng X, Wang Y, et al. Atg5-mediated autophagy deficiency in proximal tubules promotes cell cycle G2/M arrest and renal fibrosis. Autophagy. 2016; 12(9):1472-86.

55. Hartleben B, Godel M, Meyer-Schwesinger C, et al. Autophagy influences glomerular disease susceptibility and maintains podocyte homeostasis in aging mice. J Clin Invest. 2010; 120(4):1084-96

56. Fang L, Zhou $\mathrm{Y}, \mathrm{Cao} \mathrm{H}$, et al. Autophagy attenuates diabetic glomerular damage through protection of hyperglycemia-induced podocyte injury. PLoS ONE. 2013 11;8(4): e60546. 